\title{
Azimuthal Anisotropy of Charged Particles at High Transverse Momenta in Pb-Pb Collisions at $\sqrt{s_{N N}}=2.76 \mathrm{TeV}$
}

\author{
S. Chatrchyan et al. ${ }^{*}$ \\ (CMS Collaboration)
}

(Received 9 April 2012; published 10 July 2012)

\begin{abstract}
The azimuthal anisotropy of charged particles in $\mathrm{Pb}-\mathrm{Pb}$ collisions at $\sqrt{s_{N N}}=2.76 \mathrm{TeV}$ is measured with the CMS detector at the LHC over an extended transverse momentum $\left(p_{\mathrm{T}}\right)$ range up to approximately $60 \mathrm{GeV} / c$. The data cover both the low- $p_{\mathrm{T}}$ region associated with hydrodynamic flow phenomena and the high- $p_{\mathrm{T}}$ region where the anisotropies may reflect the path-length dependence of parton energy loss in the created medium. The anisotropy parameter $\left(v_{2}\right)$ of the particles is extracted by correlating charged tracks with respect to the event-plane reconstructed by using the energy deposited in forward-angle calorimeters. For the six bins of collision centrality studied, spanning the range of 0-60\% most-central events, the observed $v_{2}$ values are found to first increase with $p_{\mathrm{T}}$, reaching a maximum around $p_{\mathrm{T}}=3 \mathrm{GeV} / c$, and then to gradually decrease to almost zero, with the decline persisting up to at least $p_{\mathrm{T}}=40 \mathrm{GeV} / c$ over the full centrality range measured.
\end{abstract}

DOI: 10.1103/PhysRevLett.109.022301

PACS numbers: 25.75.Gz, 25.75.Ag, 25.75.Ld

The experiments at the Relativistic Heavy Ion Collider have provided evidence for the formation of a strongly coupled quantum chromodynamics (QCD) state of matter in ultrarelativistic nucleus-nucleus interactions [1-4]. The opaqueness of this matter to high-energy partons leads to a "jet-quenching" phenomenon where the final-state particle yield at high transverse momentum $\left(p_{\mathrm{T}}\right)$ is found to be suppressed compared to that expected from the scaling of $p p$ collision yields [1-6]. A large energy loss for partons traversing the dense QCD medium is also suggested by the recent observation of a large momentum imbalance of reconstructed back-to-back jets [7-9] in $\mathrm{Pb}-\mathrm{Pb}$ collisions at the Large Hadron Collider (LHC).

Despite the progress made on the theoretical description of jet quenching in the past decade [10], some of its key properties, such as the detailed path-length dependence of parton energy loss, remain unknown. In addition to measurements of hadron-yield suppression, observables such as the azimuthal anisotropy of high- $p_{\mathrm{T}}$ hadrons are needed to differentiate between the theoretical approaches [11-16]. The anisotropy can be characterized by the second-order Fourier harmonic coefficient $\left(v_{2}\right)$ in the azimuthal angle $(\phi)$ distribution of the hadron yield, $d N / d \phi \propto 1+2 v_{2} \cos \left[2\left(\phi-\Psi_{\mathrm{PP}}\right)\right]$, where $\Psi_{\mathrm{PP}}$ is the event-by-event azimuthal angle of the "participant plane." In a noncentral heavy-ion collision, the overlap region of the two colliding nuclei has a lenticular shape, and the interacting nucleons in this region are known as

*Full author list given at the end of the article.

Published by the American Physical Society under the terms of the Creative Commons Attribution 3.0 License. Further distribution of this work must maintain attribution to the author(s) and the published article's title, journal citation, and DOI. "participants." The participant plane is defined by the beam direction and the short direction of the lenticular region. In general, the participant plane will not contain the reaction impact parameter vector because of fluctuations that arise from having a finite number of nucleons. For high- $p_{\mathrm{T}}$ particles, an azimuthal anisotropy can be induced if there is stronger suppression of the hadron yield along the long axis than the short axis of the overlap region. The importance of jet-quenching measurements taking into account the jet orientation relative to the geometry of the interaction region was first demonstrated by the PHENIX experiment [17], where the azimuthal anisotropy of high- $p_{\mathrm{T}}$ neutral pions $\left(\pi^{0}\right)$ was studied up to $p_{\mathrm{T}} \approx$ $18 \mathrm{GeV} / c$ in AuAu collisions at $\sqrt{s_{N N}}=200 \mathrm{GeV}$.

This Letter presents a study of the azimuthal anisotropy extended to very high $p_{\mathrm{T}}$ (up to $p_{\mathrm{T}} \approx 60 \mathrm{GeV} / c$ ) for $\mathrm{Pb}-\mathrm{Pb}$ collisions at $\sqrt{s_{N N}}=2.76 \mathrm{TeV}$ at the LHC using the Compact Muon Solenoid (CMS) detector. The $v_{2}$ coefficient is determined as a function of charged particle $p_{\mathrm{T}}$ and overlap of the colliding nuclei (centrality) in the pseudorapidity regions of $|\eta|<1$ and $1<|\eta|<2$, where $\eta=-\ln [\tan (\theta / 2)]$ and $\theta$ is the polar angle relative to the counterclockwise beam direction. In the low momentum region (below a few $\mathrm{GeV} / c$ ) , $v_{2}$ is generally associated with hydrodynamic flow [18], as distinct from the jet energy-loss mechanism believed to dominate at high $p_{\mathrm{T}}$ (e.g., above $10 \mathrm{GeV} / c$ ). By using a single-track high-level trigger, a significantly larger event sample of high- $p_{\mathrm{T}}$ tracks than previously available is obtained, providing the first precise measurement of the $v_{2}$ coefficient above $20 \mathrm{GeV} / c$ in heavy-ion collisions. Our results may impose quantitative constraints on models of the in-medium energy loss of high- $p_{\mathrm{T}}$ partons, particularly the influence of the path length and the shape of the interaction region on the energy loss. 
The data used in this analysis correspond to an integrated luminosity of $150 \mu \mathrm{b}^{-1}$ and were recorded during the 2011 LHC heavy-ion running period. A detailed description of the CMS detector can be found in Ref. [19]. Its central feature is a superconducting solenoid of $6 \mathrm{~m}$ internal diameter, providing a magnetic field of $3.8 \mathrm{~T}$. Within the field volume are the silicon pixel and strip tracker, the crystal electromagnetic calorimeter, and the brass and scintillator hadron calorimeter. In $\mathrm{Pb}-\mathrm{Pb}$ collisions, trajectories of charged particles with $p_{\mathrm{T}}>200 \mathrm{MeV} / c$ are reconstructed in the silicon tracker covering the pseudorapidity region $|\eta|<2.5$, with a track momentum resolution of about $1 \%$ at $p_{\mathrm{T}}=100 \mathrm{GeV} / c$. In addition, CMS has extensive forward calorimetry, in particular, two steel and quartz-fiber Čerenkov hadronic forward (HF) calorimeters, which cover the pseudorapidity range $2.9<|\eta|<5.2$. The HF calorimeters are segmented into towers, each of which is a two-dimensional cell with a granularity of 0.5 unit in $\eta$ and $0.349 \mathrm{rad}$ in $\phi$.

Minimum bias $\mathrm{Pb}-\mathrm{Pb}$ events were triggered by coincident signals from both ends of the detector in either the beam scintillator counters at $3.23<|\eta|<4.65$ or in the HF calorimeters. Events due to noise, cosmic rays, out-oftime triggers, and beam backgrounds were suppressed by requiring a coincidence of the minimum bias trigger with bunches colliding in the interaction region. The trigger has an acceptance of $(97 \pm 3) \%$ for hadronic inelastic $\mathrm{Pb}-\mathrm{Pb}$ collisions. Because of hardware limits on the data acquisition rate, only a small fraction of all minimum bias triggered events were recorded. To maximize the event sample with high- $p_{\mathrm{T}}$ particles emitted in the $\mathrm{Pb}-\mathrm{Pb}$ collisions, a dedicated high- $p_{\mathrm{T}}$ single-track trigger was implemented by using the CMS level-1 (L1) and high-level trigger system. The trajectories of charged particles from the silicon tracker were found online by using a tracking algorithm identical to those employed in the offline track reconstruction, starting with a candidate from the threelayer silicon pixel tracker having $p_{\mathrm{T}}>11 \mathrm{GeV} / c$. For $\mathrm{Pb}-$ $\mathrm{Pb}$ events with total transverse energy at L1 (L1_ETT) above $100 \mathrm{GeV}$, a trigger efficiency of above $95 \%$ relative to offline reconstructed tracks was achieved for track $p_{\mathrm{T}}$ greater than $12 \mathrm{GeV} / c$. For the events with L1_ETT less than $100 \mathrm{GeV}$, an additional requirement of a calorimeter jet at L1 with $p_{\mathrm{T}}>16 \mathrm{GeV} / c$ [8] was imposed in order to reduce the detector readout rate. This resulted in an efficiency of about $75 \%$ starting at $p_{\mathrm{T}} \approx 12 \mathrm{GeV} / c$ but increasing to almost $100 \%$ above $p_{\mathrm{T}} \approx 20 \mathrm{GeV} / c$. In this analysis, the minimum bias data sample is used for the $v_{2}$ measurement of tracks with $1<p_{\mathrm{T}}<12 \mathrm{GeV} / c$ (also $12<p_{\mathrm{T}}<20 \mathrm{GeV} / c$ for cross-check purposes), while the high- $p_{\mathrm{T}}$ track triggered sample is used in the range of $p_{\mathrm{T}}>12 \mathrm{GeV} / c$.

Events are further selected offline by requiring energy deposits in at least three towers in each of the HF calorimeters, with at least $3 \mathrm{GeV}$ of energy in each tower, and the presence of a reconstructed primary vertex containing at least two tracks. These criteria further reduce the background from single-beam interactions (e.g., beam-gas and beam-halo), cosmic muons, and large impact parameter, ultraperipheral collisions that lead to the electromagnetic breakup of one or both $\mathrm{Pb}$ nuclei [20]. The reconstructed primary vertex is required to be located within $\pm 15 \mathrm{~cm}$ of the average vertex position along the beam axis and within a radius of $0.02 \mathrm{~cm}$ in the transverse plane. The centrality of the $\mathrm{Pb}-\mathrm{Pb}$ reaction is determined by taking fractions of the total hadronic inelastic cross section, according to percentiles of the distribution of the total energy deposited in the HF calorimeters [8]. The centrality classes used in this analysis are $0-10 \%$ (most central), 10\%-20\%, $20 \%-30 \%, 30 \%-40 \%, 40 \%-50 \%$, and $50 \%-60 \%$, ordered from the highest to the lowest HF energy deposited.

The reconstruction of the primary event vertex and the trajectories of charged particles in $\mathrm{Pb}-\mathrm{Pb}$ collisions is based on signals in the silicon pixel and strip detectors and described in detail in Ref. [6]. In selecting the charged primary tracks, a set of tight quality selections were imposed to minimize the contamination from misidentified tracks. These include requirements of a relative momentum uncertainty of less than 5\%, at least 13 hits on the track, a normalized $\chi^{2}$ for the track fit of less than 0.15 times the number of hits, and transverse and longitudinal track displacements from the primary vertex position less than 3 times the sum in quadrature of the measurement uncertainties. From studies based on $\mathrm{Pb}-\mathrm{Pb}$ events simulated by using HYDJET [21] (version 1.6), the combined geometrical acceptance and reconstruction efficiency of the primary tracks reaches about $66 \%(50 \%)$ at $|\eta|<1.0$ $(1.0<|\eta|<2.0)$ for the $5 \%$ most-central $\mathrm{Pb}-\mathrm{Pb}$ events, with little dependence on $p_{\mathrm{T}}$. For the peripheral $\mathrm{Pb}-\mathrm{Pb}$ events, the efficiency is improved by up to $5 \%$, again largely independent of $p_{\mathrm{T}}$. The fraction of misidentified tracks is kept at the $1 \%-2 \%$ level at $p_{\mathrm{T}}>2 \mathrm{GeV} / c$ over almost the entire $\eta$ and centrality ranges. It increases up to $10 \%$ for very low $p_{\mathrm{T}}(\approx 1 \mathrm{GeV} / c)$ particles in the forward $(|\eta| \approx 2)$ region for the $5 \%$ most-central $\mathrm{Pb}-\mathrm{Pb}$ events.

The analysis follows closely the event-plane method described in Ref. [22]. The observed $v_{2}$ value for a given centrality and $p_{\mathrm{T}}$ range is defined by $\boldsymbol{v}_{2}^{\text {obs }}=$ $\left\langle\cos 2\left(\phi-\Psi_{2}\right)\right\rangle$, where the average is taken over all particles in all events within a centrality and $p_{\mathrm{T}}$ bin. The second-order "event-plane" angle $\Psi_{2}$ corresponds to the event-by-event azimuthal angle of maximum particle density in $\mathrm{Pb}-\mathrm{Pb}$ collisions. It is an approximation of the participant-plane angle $\Psi_{\mathrm{PP}}$, which is not directly observable. The determination of $\Psi_{2}$ uses the energy deposited in the HF calorimeters with $\Psi_{2}=\frac{1}{2} \tan ^{-1} \frac{\sum_{i} w_{i} \sin \left(2 \phi_{i}\right)}{\sum_{i} w_{i} \cos \left(2 \phi_{i}\right)}$, where the weight factor $w_{i}$ for the $i$ th tower at azimuthal angle $\phi_{i}$ is taken as the corresponding transverse energy. The sums are taken over all the towers within a slightly truncated $\eta$ range of each $\mathrm{HF}$ calorimeter coverage. For the $v_{2}$ study in 
this analysis, charged particles detected in the tracker with $\eta>0(<0)$ are correlated with an event plane found by using energy deposited in a region of the HF spanning $-5<\eta<-3(3<\eta<5)$. In this manner, a minimum $\eta$ gap of 3 units is guaranteed between particles used in the event-plane determination and those for which the $v_{2}$ value is being measured, thereby significantly reducing the effect of other correlations that might exist, such as that from dijets. This $\eta$ gap is particularly important for the high- $p_{\mathrm{T}}$ particle studies.

The resolution of the event plane depends on the centrality and is limited by the finite number of particles used in its determination. The final $v_{2}$ coefficient in the eventplane method is evaluated by dividing the observed value $v_{2}^{\text {obs }}$ by a "resolution-correction" factor $R$, with $v_{2}=$ $v_{2}^{\text {obs }} / R$ and where $R$ can range from 0 to 1 [23], with a better resolution corresponding to a larger value of $R$. An event-averaged resolution-correction factor can be found experimentally by extracting separate event-plane angles using particles emitted into three nonoverlapping $\eta$ regions. In this "three-subevent" technique, which is described in more detail in Ref. [23], the resolutioncorrection factor for a given $\eta$ region (denoted $A$, with $B$ and $C$ used for the other two $\eta$ ranges) is found by using

$$
R_{A}=\sqrt{\frac{\left\langle\cos \left[2\left(\Psi_{2}^{A}-\Psi_{2}^{B}\right)\right]\right\rangle\left\langle\cos \left[2\left(\Psi_{2}^{A}-\Psi_{2}^{C}\right)\right]\right\rangle}{\left\langle\cos \left[2\left(\Psi_{2}^{B}-\Psi_{2}^{C}\right)\right]\right\rangle}} .
$$

The averages are over all events corresponding to a given centrality bin. Reconstructed tracks with $|\eta|<0.8$ and $p_{\mathrm{T}}>1 \mathrm{GeV} / c$ are used for the "third" $\eta$ range (denoted $C$ ) introduced to determine the resolution of the event planes found by using the HF detectors at $-5<\eta<-3$ (denoted $A$ ) and $3<\eta<5$ (denoted $B$ ). In the calculation of $\Psi_{2}^{C}$, the weight factor $w_{i}$ of the $i$ th track at angle $\phi_{i}$ is taken as the corresponding transverse momentum. The extracted $R$ values for the HF event planes are found to vary between 0.55 and 0.84 , reaching a maximum value for events in the $20 \%-30 \%$ centrality bin. The difference in the resolution-correction factors found for the two HF event planes is less than $1 \%$.

The sensitivity of the deduced $v_{2}$ values to the size of the minimum $\eta$ gap is explored by defining additional event planes with different pseudorapidity ranges. Varying the gap size from 3 to 4 units, the $v_{2}$ values are found to be consistent within $\pm 2.5 \%$ (central) or $\pm 10 \%$ (peripheral). For all systematic studies, relative uncertainties to $v_{2}$ are quoted. The influence of misidentified tracks is another source of systematic uncertainty at high $p_{\mathrm{T}}$. The $v_{2}$ of misidentified tracks in data is estimated by using a very loose track selection. By taking the misidentified-track $\boldsymbol{v}_{2}$ signal, together with the probability of reconstructing a fake track as determined from simulated events, the systematic uncertainty on the observed $v_{2}$ values is estimated to be $\pm 1 \%-3 \%$, depending on $p_{\mathrm{T}}$ and centrality. Potential biases of the events triggered by the high- $p_{\mathrm{T}}$ track algorithm are investigated. A comparison of $v_{2}$ results for $12<p_{\mathrm{T}}<20 \mathrm{GeV} / c$ obtained from minimum bias and high- $p_{\mathrm{T}}$ track triggered samples shows a variation of less than $\pm 1 \%$. To account for nonuniformities in the detector acceptance that can lead to artificial asymmetries in the event-plane angle distribution and thereby also affect the deduced $v_{2}$ values, a Fourier-analysis-based "flattening" procedure is used [23] where each calculated event-plane angle is shifted slightly to recover a uniform azimuthal distribution of angles [22]. Monte Carlo studies have shown that this flattening procedure fully corrects for nonuniformities in the CMS detector response. The overall systematic uncertainties associated with the event-plane flattening procedure and the resolution-correction determination are found to be less than $\pm 1 \%$. Additional uncertainties due to track-quality requirements are examined by loosening or tightening the selection criteria described previously and are found to be less than $\pm 0.5 \%$. The different systematic sources are added in quadrature to obtain the overall systematic uncertainty in $v_{2}$.

The results of $v_{2}$, as a function of $p_{\mathrm{T}}$ from 1 to $60 \mathrm{GeV} / c$ for events with centralities ranging from $0-10 \%$ (central) to $50 \%-60 \%$ (peripheral), are presented in Fig. 1 for $|\eta|<1$. The highest $p_{\mathrm{T}}$ bin covers a range from 48.0 to $60.8 \mathrm{GeV} / c$. The new CMS results in this Letter significantly extend the $p_{\mathrm{T}}$ reach of $v_{2}$ measurements previously achieved by the ALICE [24], ATLAS [25], and CMS [22] Collaborations. The CMS and ATLAS results based on event-plane analyses using forward calorimeters for the event-plane determination show agreement within $2 \%-3 \%$ up to $p_{\mathrm{T}} \approx 20 \mathrm{GeV} / c$.

The $p_{\mathrm{T}}$ dependence of $\boldsymbol{v}_{2}$ shows a trend of rapid rise, reaching a maximum at $\approx 3 \mathrm{GeV} / c$, followed by a dramatic decrease for $p_{\mathrm{T}}$ values up to about $10 \mathrm{GeV} / c$. Beyond $p_{\mathrm{T}} \approx 10 \mathrm{GeV} / c$, the $v_{2}$ values show a much weaker dependence on $p_{\mathrm{T}}$ and gradually decrease but remain larger than zero up to at least $p_{\mathrm{T}} \approx 40 \mathrm{GeV} / c$. The magnitude of $v_{2}$ at high $p_{\mathrm{T}}$ can probe the path-length (l) dependence of parton energy loss $(\Delta E), \Delta E \sim l^{\alpha}$ [11-16]. At Relativistic Heavy Ion Collider energies, the $\pi^{0} v_{2}$ data for $p_{\mathrm{T}} \sim 6-10 \mathrm{GeV} / c$ support a scenario of $\alpha=3$ based on AdS/CFT gravity-gauge dual modeling, while the perturbative QCD calculations ( $\alpha=1$ for collisional energy loss and $\alpha=2$ for radiative energy loss) seem to underpredict the data [17]. The $v_{2}$ results reported in this Letter extend to a significantly wider $p_{\mathrm{T}}$ regime, where particle production is unambiguously dominated by parton fragmentation and energy-loss models are expected to be more applicable. The clear $p_{\mathrm{T}}$ dependence of $v_{2}$ observed at the LHC energy suggests that additional dynamical modeling of parton modification, particularly by incorporating the proper initial parton energy dependence, is required in order to further examine the path-length dependence of energy loss (i.e., $\alpha$ parameter). 


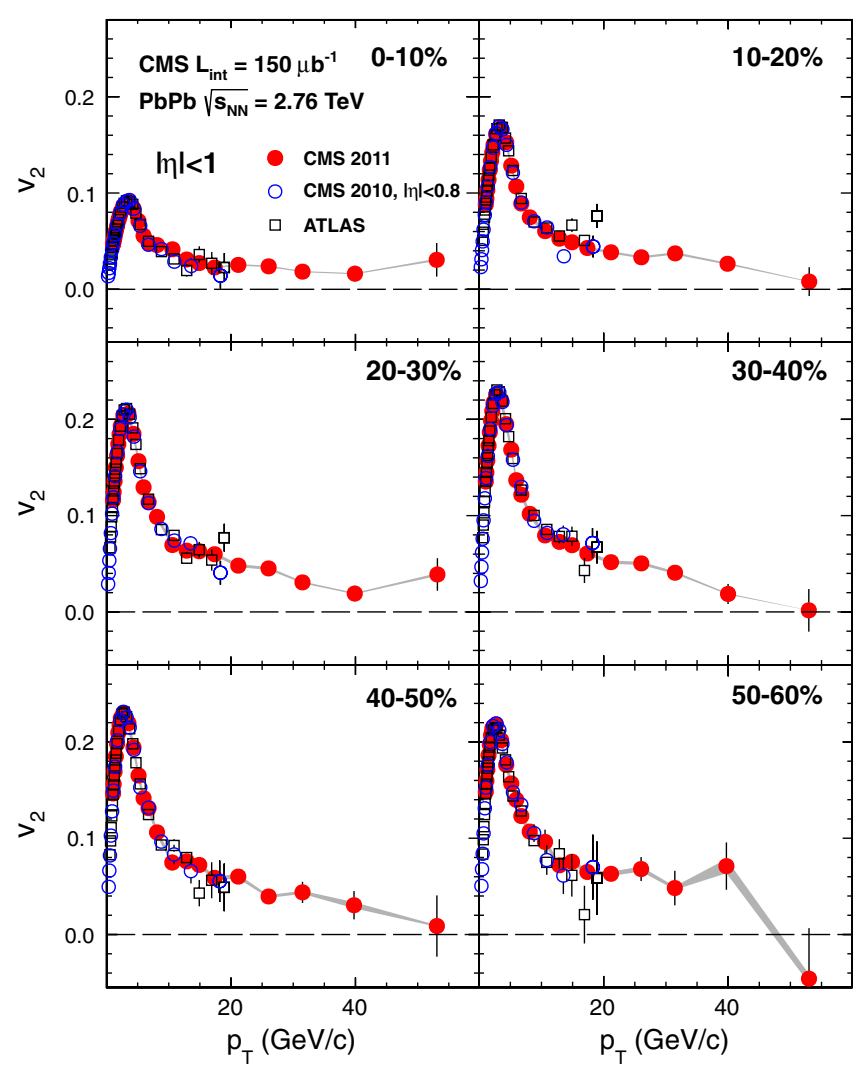

FIG. 1 (color online). The single-particle azimuthal anisotropy $v_{2}$ as a function of the charged particle transverse momentum from 1 to $60 \mathrm{GeV} / c$ with $|\eta|<1$ for six centrality ranges in $\mathrm{Pb}-\mathrm{Pb}$ collisions at $\sqrt{s_{N N}}=2.76 \mathrm{TeV}$, measured by the CMS experiment (solid markers). Error bars denote the statistical uncertainties, while the gray bands correspond to the small systematic uncertainties. Comparison to results from the ATLAS (open squares) and CMS (open circles) experiments using data collected in 2010 is also shown.

Figure 2 shows the $v_{2}$ dependences on the number of participant nucleons ( $N_{\text {part }}$ ) associated with each centrality bin through a Glauber model calculation. The corresponding participant eccentricities of the overlap region vary from 0.46 to 0.093 . Different $p_{\mathrm{T}}$ bins are shown for two pseudorapidity ranges: $|\eta|<1$ and $1<|\eta|<2$. The results appear to be independent of $\eta$ in all $p_{\mathrm{T}}$ bins within the statistical uncertainties. Also, the $v_{2}$ values tend to decrease with increasing collision centrality (i.e., larger $N_{\text {part }}$ ) over a wide $p_{\mathrm{T}}$ range (although this trend appears to be reversed for $3.2<p_{\mathrm{T}}<4.0 \mathrm{GeV} / c$ toward very peripheral events). This behavior is expected for low- $p_{\mathrm{T}}$ (below a few $\mathrm{GeV} / c$ ) particles in the context of the relationship between hydrodynamic flow phenomena and the eccentricity of the initial-state collision geometry. The similar trend observed for very high- $p_{\mathrm{T}}$ particles, at least up to $p_{\mathrm{T}} \approx 48 \mathrm{GeV} / c$, reflects how the $v_{2}$ results at high $p_{\mathrm{T}}$ are also sensitive to the initial geometry. This indicates that the initial conditions of the hot QCD medium can be further constrained by simultaneously comparing data with

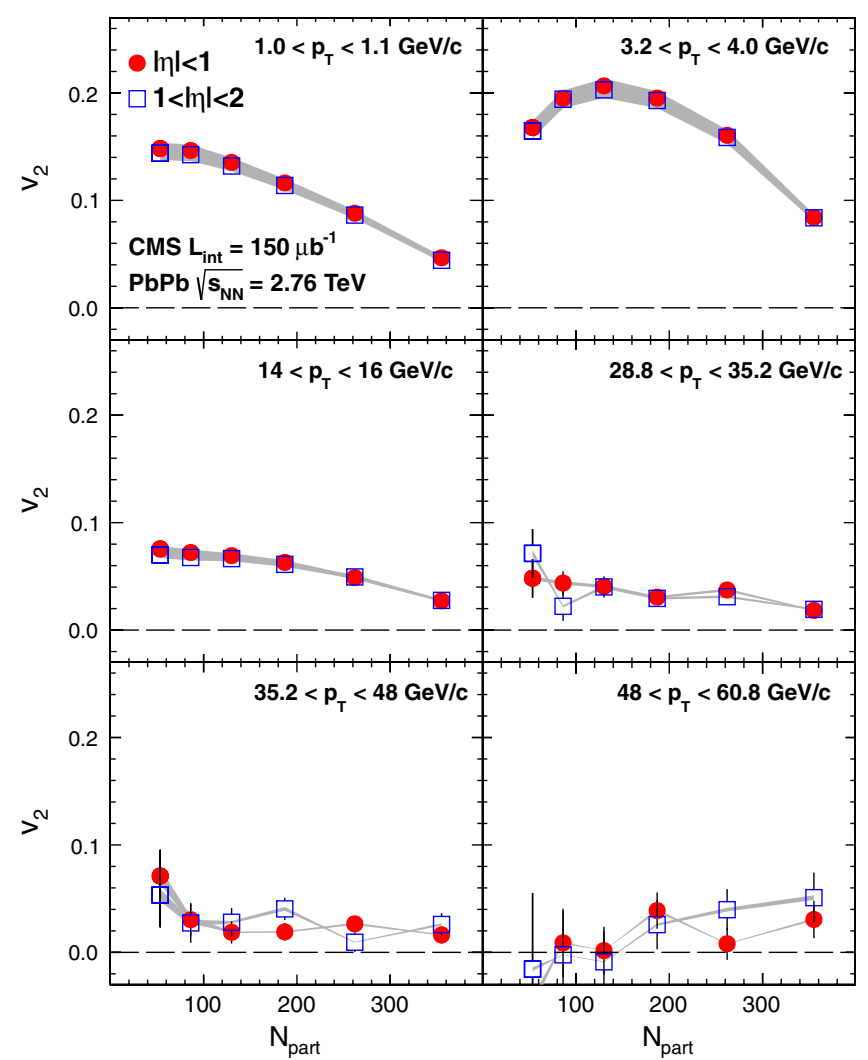

FIG. 2 (color online). The single-particle azimuthal anisotropy $v_{2}$ as a function of the number of participating nucleons with $|\eta|<1$ (solid circles) and $1<|\eta|<2$ (open squares) for six selected $p_{\mathrm{T}}$ ranges in $\mathrm{Pb}-\mathrm{Pb}$ collisions at $\sqrt{s_{N N}}=2.76 \mathrm{TeV}$, measured by the CMS experiment. Error bars denote the statistical uncertainties, while the gray bands correspond to the systematic uncertainties.

theoretical calculations from both hydrodynamics at low $p_{\mathrm{T}}$ and in-medium parton energy loss at high $p_{\mathrm{T}}$.

In summary, the azimuthal anisotropy of charged particles with respect to the event plane has been studied in $\mathrm{Pb}$ $\mathrm{Pb}$ collisions at $\sqrt{s_{N N}}=2.76 \mathrm{TeV}$ using the CMS detector. The $v_{2}$ coefficient was determined over a wide range in $p_{\mathrm{T}}$ up to approximately $60 \mathrm{GeV} / c$, as a function of collision centrality. The results reported in this Letter significantly improve the statistical precision of previous $v_{2}$ measurements for $12<p_{\mathrm{T}}<20 \mathrm{GeV} / c$ and explore for the first time the very high- $p_{\mathrm{T}}$ region beyond $20 \mathrm{GeV} / c$. The $v_{2}\left(p_{\mathrm{T}}\right)$ behavior shows a trend of rapid rise to a maximum at $p_{\mathrm{T}} \approx 3 \mathrm{GeV} / c$ and a subsequent fall for all centrality and $|\eta|$ ranges. Beyond $p_{\mathrm{T}} \approx 10 \mathrm{GeV} / c$, the observed $v_{2}$ values still show a clear $p_{\mathrm{T}}$ dependence but with a more moderate decrease with $p_{\mathrm{T}}$, remaining finite up to at least $p_{\mathrm{T}} \approx 40 \mathrm{GeV} / c$. A common trend in the centrality dependence of $v_{2}$ is observed for particles over a wide range of $p_{\mathrm{T}}$ up to approximately $48 \mathrm{GeV} / c$, suggesting a potential connection to the initial-state geometry. The precision data over a wide kinematic range presented here will provide important constraints on models of parton energy loss, 
particularly in terms of its dependence on the initial conditions, parton energy, and path length through the medium.

We congratulate our colleagues in the CERN accelerator departments for the excellent performance of the LHC machine. We thank the technical and administrative staff at CERN and other CMS institutes and acknowledge support from FMSR (Austria); FNRS and FWO (Belgium); CNPq, CAPES, FAPERJ, and FAPESP (Brazil); MES (Bulgaria); CERN; CAS, MoST, and NSFC (China); COLCIENCIAS (Colombia); MSES (Croatia); RPF (Cyprus); Academy of Sciences and NICPB (Estonia); Academy of Finland, MEC, and HIP (Finland); CEA and CNRS/IN2P3 (France); BMBF, DFG, and HGF (Germany); GSRT (Greece); OTKA and NKTH (Hungary); DAE and DST (India); IPM (Iran); SFI (Ireland); INFN (Italy); NRF and WCU (Korea); LAS (Lithuania); CINVESTAV, CONACYT, SEP, and UASLP-FAI (Mexico); MSI (New Zealand); PAEC (Pakistan); SCSR (Poland); FCT (Portugal); JINR (Armenia, Belarus, Georgia, Ukraine, Uzbekistan); MON, RosAtom, RAS, and RFBR (Russia); MSTD (Serbia); MICINN and CPAN (Spain); Swiss Funding Agencies (Switzerland); NSC (Taipei); TUBITAK and TAEK (Turkey); STFC (United Kingdom); DOE and NSF (USA).

[1] I. Arsene et al. (BRAHMS Collaboration), Nucl. Phys. A757, 1 (2005).

[2] K. Adcox et al. (PHENIX Collaboration), Nucl. Phys. A757, 184 (2005).

[3] B. Back et al. (PHOBOS Collaboration), Nucl. Phys. A757, 28 (2005).

[4] J. Adams et al. (STAR Collaboration), Nucl. Phys. A757, 102 (2005)
[5] K. Aamodt et al. (ALICE Collaboration), Phys. Lett. B 696, 30 (2011).

[6] S. Chatrchyan et al. (CMS Collaboration), Eur. Phys. J. C 72, 1945 (2012).

[7] G. Aad et al. (ATLAS Collaboration), Phys. Rev. Lett. 105, 252303 (2010).

[8] S. Chatrchyan et al. (CMS Collaboration), Phys. Rev. C 84, 024906 (2011).

[9] S. Chatrchyan et al. (CMS Collaboration), Phys. Lett. B 712, 176 (2012).

[10] A. Majumder and M. Van Leeuwen, Prog. Part. Nucl. Phys. 66, 41 (2011).

[11] S. Peigne and A. V. Smilga, Phys. Usp. 52, 659 (2009).

[12] S. Wicks, W. Horowitz, M. Djordjevic, and M. Gyulassy, Nucl. Phys. A784, 426 (2007).

[13] J. Jia and R. Wei, Phys. Rev. C 82, 024902 (2010).

[14] J. Jia, W. Horowitz, and J. Liao, Phys. Rev. C 84, 034904 (2011).

[15] T. Renk, Phys. Rev. C 83, 024908 (2011).

[16] B. Betz, M. Gyulassy, and G. Torrieri, Phys. Rev. C 84, 024913 (2011).

[17] A. Adare et al. (PHENIX Collaboration), Phys. Rev. Lett. 105, 142301 (2010).

[18] S. A. Voloshin, A. M. Poskanzer, and R. Snellings, arXiv:0809.2949.

[19] S. Chatrchyan et al. (CMS Collaboration), JINST 3, S08004 (2008).

[20] O. Djuvsland and J. Nystrand, Phys. Rev. C 83, 041901 (2011).

[21] I. P. Lokhtin and A. M. Snigirev, Eur. Phys. J. C 45, 211 (2006).

[22] CMS Collaboration, arXiv:1204.1409 [Phys. Rev. C (to be published)].

[23] A. M. Poskanzer and S. A. Voloshin, Phys. Rev. C 58, 1671 (1998)

[24] K. Aamodt et al. (ALICE Collaboration), Phys. Rev. Lett. 105, 252302 (2010).

[25] G. Aad et al. (ATLAS Collaboration), Phys. Lett. B 707, 330 (2012).

S. Chatrchyan, ${ }^{1}$ V. Khachatryan, ${ }^{1}$ A. M. Sirunyan, ${ }^{1}$ A. Tumasyan, ${ }^{1}$ W. Adam, ${ }^{2}$ T. Bergauer, ${ }^{2}$ M. Dragicevic, ${ }^{2}$ J. Erö, ${ }^{2}$ C. Fabjan, ${ }^{2}$ M. Friedl, ${ }^{2}$ R. Frühwirth, ${ }^{2}$ V. M. Ghete, ${ }^{2}$ J. Hammer, ${ }^{2, b}$ N. Hörmann, ${ }^{2}$ J. Hrubec, ${ }^{2}$ M. Jeitler, ${ }^{2}$

W. Kiesenhofer, ${ }^{2}$ M. Krammer, ${ }^{2}$ D. Liko, ${ }^{2}$ I. Mikulec, ${ }^{2}$ M. Pernicka, ${ }^{2, a}$ B. Rahbaran, ${ }^{2}$ C. Rohringer, ${ }^{2}$ H. Rohringer, ${ }^{2}$ R. Schöfbeck, ${ }^{2}$ J. Strauss, ${ }^{2}$ A. Taurok,${ }^{2}$ F. Teischinger, ${ }^{2}$ P. Wagner, ${ }^{2}$ W. Waltenberger, ${ }^{2}$ G. Walzel, ${ }^{2}$ E. Widl, ${ }^{2}$ C.-E. Wulz, ${ }^{2}$ V. Mossolov, ${ }^{3}$ N. Shumeiko, ${ }^{3}$ J. Suarez Gonzalez, ${ }^{3}$ S. Bansal, ${ }^{4}$ K. Cerny, ${ }^{4}$ T. Cornelis, ${ }^{4}$ E. A. De Wolf, ${ }^{4}$ X. Janssen, ${ }^{4}$ S. Luyckx, ${ }^{4}$ T. Maes, ${ }^{4}$ L. Mucibello, ${ }^{4}$ S. Ochesanu, ${ }^{4}$ B. Roland,${ }^{4}$ R. Rougny, ${ }^{4}$ M. Selvaggi, ${ }^{4}$ H. Van Haevermaet, ${ }^{4}$ P. Van Mechelen, ${ }^{4}$ N. Van Remortel, ${ }^{4}$ A. Van Spilbeeck, ${ }^{4}$ F. Blekman, ${ }^{5}$ S. Blyweert, ${ }^{5}$

J. D'Hondt, ${ }^{5}$ R. Gonzalez Suarez, ${ }^{5}$ A. Kalogeropoulos, ${ }^{5}$ M. Maes, ${ }^{5}$ A. Olbrechts, ${ }^{5}$ W. Van Doninck, ${ }^{5}$

P. Van Mulders, ${ }^{5}$ G. P. Van Onsem, ${ }^{5}$ I. Villella, ${ }^{5}$ O. Charaf,${ }^{6}$ B. Clerbaux, ${ }^{6}$ G. De Lentdecker, ${ }^{6}$ V. Dero, ${ }^{6}$ A. P. R. Gay, ${ }^{6}$ T. Hreus, ${ }^{6}$ A. Léonard, ${ }^{6}$ P. E. Marage, ${ }^{6}$ T. Reis, ${ }^{6}$ L. Thomas, ${ }^{6}$ C. Vander Velde, ${ }^{6}$ P. Vanlaer, ${ }^{6}$ V. Adler, ${ }^{7}$ K. Beernaert, ${ }^{7}$ A. Cimmino, ${ }^{7}$ S. Costantini, ${ }^{7}$ G. Garcia, ${ }^{7}$ M. Grunewald, ${ }^{7}$ B. Klein, ${ }^{7}$ J. Lellouch, ${ }^{7}$ A. Marinov, ${ }^{7}$ J. Mccartin, ${ }^{7}$

A. A. Ocampo Rios, ${ }^{7}$ D. Ryckbosch,${ }^{7}$ N. Strobbe, ${ }^{7}$ F. Thyssen, ${ }^{7}$ M. Tytgat, ${ }^{7}$ L. Vanelderen, ${ }^{7}$ P. Verwilligen, ${ }^{7}$ S. Walsh ${ }^{7}$ E. Yazgan, ${ }^{7}$ N. Zaganidis, ${ }^{7}$ S. Basegmez, ${ }^{8}$ G. Bruno, ${ }^{8}$ L. Ceard, ${ }^{8}$ C. Delaere, ${ }^{8}$ T. du Pree, ${ }^{8}$ D. Favart, ${ }^{8}$ L. Forthomme, ${ }^{8}$ A. Giammanco, ${ }^{8, \mathrm{c}}$ J. Hollar, ${ }^{8}$ V. Lemaitre, ${ }^{8}$ J. Liao, ${ }^{8}$ O. Militaru, ${ }^{8}$ C. Nuttens, ${ }^{8}$ D. Pagano,${ }^{8}$ A. Pin, ${ }^{8}$ K. Piotrzkowski, ${ }^{8}$ N. Schul, ${ }^{8}$ N. Beliy, ${ }^{9}$ T. Caebergs,,${ }^{9}$ E. Daubie, ${ }^{9}$ G. H. Hammad, ${ }^{9}$ G. A. Alves,${ }^{10}$ M. Correa Martins Junior, ${ }^{10}$ D. De Jesus Damiao, ${ }^{10}$ T. Martins, ${ }^{10}$ M. E. Pol,${ }^{10}$ M. H. G. Souza, ${ }^{10}$ W. L. Aldá Júnior,${ }^{11}$ 
W. Carvalho, ${ }^{11}$ A. Custódio, ${ }^{11}$ E. M. Da Costa,${ }^{11}$ C. De Oliveira Martins, ${ }^{11}$ S. Fonseca De Souza, ${ }^{11}$

D. Matos Figueiredo, ${ }^{11}$ L. Mundim, ${ }^{11}$ H. Nogima, ${ }^{11}$ V. Oguri, ${ }^{11}$ W. L. Prado Da Silva, ${ }^{11}$ A. Santoro, ${ }^{11}$ S. M. Silva Do Amaral, ${ }^{11}$ L. Soares Jorge, ${ }^{11}$ A. Sznajder, ${ }^{11}$ T. S. Anjos, ${ }^{12, d}$ C. A. Bernardes, ${ }^{12, d}$ F. A. Dias, ${ }^{12, e}$ T. R. Fernandez Perez Tomei, ${ }^{12}$ E. M. Gregores,${ }^{12, d}$ C. Lagana, ${ }^{12}$ F. Marinho, ${ }^{12}$ P. G. Mercadante, ${ }^{12, d}$ S. F. Novaes,${ }^{12}$ Sandra S. Padula, ${ }^{12}$ V. Genchev, ${ }^{13, b}$ P. Iaydjiev, ${ }^{13, b}$ S. Piperov, ${ }^{13}$ M. Rodozov, ${ }^{13}$ S. Stoykova, ${ }^{13}$ G. Sultanov, ${ }^{13}$ V. Tcholakov, ${ }^{13}$ R. Trayanov, ${ }^{13}$ M. Vutova, ${ }^{13}$ A. Dimitrov, ${ }^{14}$ R. Hadjiiska,,${ }^{14}$ V. Kozhuharov, ${ }^{14}$ L. Litov, ${ }^{14}$ B. Pavlov, ${ }^{14}$ P. Petkov, ${ }^{14}$ J. G. Bian, ${ }^{15}$ G. M. Chen, ${ }^{15}$ H. S. Chen, ${ }^{15}$ C. H. Jiang, ${ }^{15}$ D. Liang, ${ }^{15}$ S. Liang, ${ }^{15}$ X. Meng, ${ }^{15}$ J. Tao, ${ }^{15}$ J. Wang, ${ }^{15} \mathrm{~J}$. Wang, ${ }^{15} \mathrm{X}$. Wang, ${ }^{15} \mathrm{Z}$. Wang, ${ }^{15} \mathrm{H}$. Xiao, ${ }^{15} \mathrm{M}$. Xu, ${ }^{15} \mathrm{~J}$. Zang, ${ }^{15} \mathrm{Z}$. Zhang, ${ }^{15} \mathrm{C}$. Asawatangtrakuldee, ${ }^{16}$ Y. Ban, ${ }^{16}$ S. Guo, ${ }^{16}$ Y. Guo, ${ }^{16}$ W. Li, ${ }^{16}$ S. Liu, ${ }^{16}$ Y. Mao, ${ }^{16}$ S. J. Qian, ${ }^{16}$ H. Teng, ${ }^{16}$ S. Wang, ${ }^{16}$ B. Zhu, ${ }^{16}$ W. Zou ${ }^{16}$ C. Avila, ${ }^{17}$ B. Gomez Moreno, ${ }^{17}$ A. F. Osorio Oliveros,${ }^{17}$ J. C. Sanabria, ${ }^{17}$ N. Godinovic, ${ }^{18}$ D. Lelas,${ }^{18}$ R. Plestina,${ }^{18, f}$ D. Polic, ${ }^{18}$ I. Puljak, ${ }^{18, b}$ Z. Antunovic, ${ }^{19}$ M. Dzelalija, ${ }^{19}$ M. Kovac, ${ }^{19}$ V. Brigljevic, ${ }^{20}$ S. Duric, ${ }^{20}$ K. Kadija, ${ }^{20}$ J. Luetic,${ }^{20}$ S. Morovic,${ }^{20}$ A. Attikis,${ }^{21}$ M. Galanti, ${ }^{21}$ G. Mavromanolakis, ${ }^{21}$ J. Mousa, ${ }^{21}$ C. Nicolaou, ${ }^{21}$ F. Ptochos, ${ }^{21}$ P. A. Razis, ${ }^{21}$ M. Finger, ${ }^{22}$ M. Finger, Jr., ${ }^{22}$ Y. Assran, ${ }^{23, \mathrm{~g}}$ S. Elgammal, ${ }^{23}$ A. Ellithi Kamel,${ }^{23, \mathrm{~h}}$ S. Khalil, ${ }^{23, \mathrm{i}}$ M. A. Mahmoud, ${ }^{23, j}$ A. Radi, ${ }^{23}$ M. Kadastik, ${ }^{24}$ M. Müntel, ${ }^{24}$ M. Raidal, ${ }^{24}$ L. Rebane, ${ }^{24}$ A. Tiko, ${ }^{24}$ V. Azzolini, ${ }^{25}$ P. Eerola ${ }^{25}$ G. Fedi, ${ }^{25}$ M. Voutilainen, ${ }^{25}$ J. Härkönen, ${ }^{26}$ A. Heikkinen, ${ }^{26}$ V. Karimäki, ${ }^{26}$ R. Kinnunen, ${ }^{26}$ M. J. Kortelainen, ${ }^{26}$ T. Lampén, ${ }^{26}$ K. Lassila-Perini, ${ }^{26} \mathrm{~S}$. Lehti, ${ }^{26}$ T. Lindén, ${ }^{26}$ P. Luukka, ${ }^{26}$ T. Mäenpääa, ${ }^{26}$ T. Peltola, ${ }^{26}$ E. Tuominen, ${ }^{26}$ J. Tuominiemi, ${ }^{26}$ E. Tuovinen, ${ }^{26}$ D. Ungaro, ${ }^{26}$ L. Wendland, ${ }^{26} \mathrm{~K}$. Banzuzi, ${ }^{27}$ A. Korpela, ${ }^{27}$ T. Tuuva, ${ }^{27}$ M. Besancon, ${ }^{28}$ S. Choudhury, ${ }^{28}$ M. Dejardin,${ }^{28}$ D. Denegri, ${ }^{28}$ B. Fabbro, ${ }^{28}$ J. L. Faure, ${ }^{28}$ F. Ferri ${ }^{28}$ S. Ganjour, ${ }^{28}$ A. Givernaud ${ }^{28}$ P. Gras, ${ }^{28}$ G. Hamel de Monchenault ${ }^{28}{ }^{28}$. Jarry, ${ }^{28}$ E. Locci, ${ }^{28}$ J. Malcles,${ }^{28}$ L. Millischer, ${ }^{28}$ A. Nayak, ${ }^{28}$ J. Rander, ${ }^{28}$ A. Rosowsky, ${ }^{28}$ I. Shreyber, ${ }^{28}$ M. Titov,${ }^{28}$ S. Baffioni, ${ }^{29}$ F. Beaudette,${ }^{29}$ L. Benhabib, ${ }^{29}$ L. Bianchini, ${ }^{29}$ M. Bluj,${ }^{29, k}$ C. Broutin, ${ }^{29}$ P. Busson, ${ }^{29}$ C. Charlot,${ }^{29}$ N. Daci, ${ }^{29}$ T. Dahms,${ }^{29}$ L. Dobrzynski, ${ }^{29}$ R. Granier de Cassagnac, ${ }^{29}$ M. Haguenauer ${ }^{29}$ P. Miné, ${ }^{29}$ C. Mironov, ${ }^{29}$ C. Ochando, ${ }^{29}$ P. Paganini, ${ }^{29}$ D. Sabes,${ }^{29}$ R. Salerno, ${ }^{29}$ Y. Sirois,${ }^{29}$ C. Veelken, ${ }^{29}$ A. Zabi,${ }^{29}$ J.-L. Agram,${ }^{30,1}$ J. Andrea,${ }^{30}$ D. Bloch, ${ }^{30}$ D. Bodin, ${ }^{30}$ J.-M. Brom,${ }^{30}$ M. Cardaci, ${ }^{30}$ E. C. Chabert, ${ }^{30}$ C. Collard,${ }^{30}$ E. Conte,${ }^{30,1}$ F. Drouhin, ${ }^{30,1}$ C. Ferro, ${ }^{30}$ J.-C. Fontaine, ${ }^{30,1}$ D. Gelé, ${ }^{30}$ U. Goerlach, ${ }^{30}$ P. Juillot, ${ }^{30}$ M. Karim, ${ }^{30,1}$ A.-C. Le Bihan, ${ }^{30}$ P. Van Hove, ${ }^{30}$ F. Fassi ${ }^{31}$ D. Mercier, ${ }^{31}$ S. Beauceron, ${ }^{32}$ N. Beaupere, ${ }^{32}$ O. Bondu, ${ }^{32}$ G. Boudoul, ${ }^{32}$ H. Brun, ${ }^{32}$ J. Chasserat, ${ }^{32}$ R. Chierici, ${ }^{32, b}$ D. Contardo, ${ }^{32}$ P. Depasse, ${ }^{32}$ H. El Mamouni, ${ }^{32}$ J. Fay, ${ }^{32}$ S. Gascon, ${ }^{32}$ M. Gouzevitch, ${ }^{32}$ B. Ille, ${ }^{32}$ T. Kurca ${ }^{32}$ M. Lethuillier, ${ }^{32}$ L. Mirabito, ${ }^{32}$ S. Perries, ${ }^{32}$ V. Sordini, ${ }^{32}$ S. Tosi, ${ }^{32}$ Y. Tschudi, ${ }^{32}$ P. Verdier, ${ }^{32}$ S. Viret,${ }^{32}$ Z. Tsamalaidze, ${ }^{33}$ G. Anagnostou, ${ }^{34}$ S. Beranek, ${ }^{34}$ M. Edelhoff,,${ }^{34}$ L. Feld,${ }^{34}$ N. Heracleous, ${ }^{34}$ O. Hindrichs,${ }^{34}$ R. Jussen, ${ }^{34}$ K. Klein, ${ }^{34}$ J. Merz, ${ }^{34}$ A. Ostapchuk,${ }^{34}$ A. Perieanu, ${ }^{34}$ F. Raupach,${ }^{34}$ J. Sammet, ${ }^{34}$ S. Schael, ${ }^{34}$ D. Sprenger ${ }^{34}$ H. Weber, ${ }^{34}$ B. Wittmer, ${ }^{34}$ V. Zhukov, ${ }^{34, m}$ M. Ata,${ }^{35}$ J. Caudron, ${ }^{35}$ E. Dietz-Laursonn, ${ }^{35}$ D. Duchardt, ${ }^{35}$ M. Erdmann, ${ }^{35}$ A. Güth, ${ }^{35}$ T. Hebbeker, ${ }^{35}$ C. Heidemann, ${ }^{35}$ K. Hoepfner, ${ }^{35}$ T. Klimkovich, ${ }^{35}$ D. Klingebiel,${ }^{35}$ P. Kreuzer, ${ }^{35}$ D. Lanske, ${ }^{35, a}$ J. Lingemann, ${ }^{35}$ C. Magass, ${ }^{35}$ M. Merschmeyer, ${ }^{35}$ A. Meyer,,${ }^{35}$ M. Olschewski ${ }^{35}$ P. Papacz, ${ }^{35}$ H. Pieta, ${ }^{35}$ H. Reithler, ${ }^{35}$ S. A. Schmitz ${ }^{35}$ L. Sonnenschein, ${ }^{35}$ J. Steggemann, ${ }^{35}$

D. Teyssier, ${ }^{35} \mathrm{M}$. Weber, ${ }^{35} \mathrm{M}$. Bontenackels, ${ }^{36} \mathrm{~V}$. Cherepanov, ${ }^{36} \mathrm{M}$. Davids, ${ }^{36} \mathrm{G}$. Flügge, ${ }^{36} \mathrm{H}$. Geenen, ${ }^{36}$ M. Geisler, ${ }^{36}$ W. Haj Ahmad, ${ }^{36}$ F. Hoehle, ${ }^{36}$ B. Kargoll, ${ }^{36}$ T. Kress, ${ }^{36}$ Y. Kuessel, ${ }^{36}$ A. Linn, ${ }^{36}$ A. Nowack, ${ }^{36}$ L. Perchalla, ${ }^{36}$ O. Pooth, ${ }^{36}$ J. Rennefeld ${ }^{36}$ P. Sauerland, ${ }^{36}$ A. Stahl,${ }^{36}$ M. Aldaya Martin, ${ }^{37}$ J. Behr, ${ }^{37}$ W. Behrenhoff, ${ }^{37}$ U. Behrens, ${ }^{37}$ M. Bergholz,${ }^{37, n}$ A. Bethani, ${ }^{37}$ K. Borras,${ }^{37}$ A. Burgmeier, ${ }^{37}$ A. Cakir,,${ }^{37}$ L. Calligaris, ${ }^{37}$ A. Campbell,${ }^{37}$ E. Castro,,${ }^{37}$ F. Costanza, ${ }^{37}$ D. Dammann,${ }^{37}$ G. Eckerlin, ${ }^{37}$ D. Eckstein, ${ }^{37}$ D. Fischer, ${ }^{37}$ G. Flucke, ${ }^{37}$ A. Geiser,${ }^{37}$ I. Glushkov, ${ }^{37}$ S. Habib,${ }^{37}$ J. Hauk,${ }^{37}$ H. Jung,${ }^{37, b}$ M. Kasemann,${ }^{37}$ P. Katsas, ${ }^{37}$ C. Kleinwort, ${ }^{37}$ H. Kluge, ${ }^{37}$ A. Knutsson, ${ }^{37}$ M. Krämer, ${ }^{37}$ D. Krücker, ${ }^{37}$ E. Kuznetsova, ${ }^{37}$ W. Lange,${ }^{37}$ W. Lohmann, ${ }^{37, n}$ B. Lutz, ${ }^{37}$ R. Mankel, ${ }^{37}$ I. Marfin, ${ }^{37}$ M. Marienfeld, ${ }^{37}$ I.-A. Melzer-Pellmann, ${ }^{37}$ A. B. Meyer, ${ }^{37}$ J. Mnich, ${ }^{37}$ A. Mussgiller, ${ }^{37}$ S. Naumann-Emme, ${ }^{37}$ J. Olzem,${ }^{37}$ H. Perrey, ${ }^{37}$ A. Petrukhin,,${ }^{37}$ D. Pitzl,${ }^{37}$ A. Raspereza, ${ }^{37}$ P. M. Ribeiro Cipriano, ${ }^{37}$ C. Riedl, ${ }^{37}$ M. Rosin, ${ }^{37}$ J. Salfeld-Nebgen, ${ }^{37}$ R. Schmidt, ${ }^{37, n}$ T. Schoerner-Sadenius, ${ }^{37}$ N. Sen,${ }^{37}$ A. Spiridonov, ${ }^{37}$ M. Stein, ${ }^{37}$ R. Walsh,${ }^{37}$ C. Wissing, ${ }^{37}$ C. Autermann, ${ }^{38}$ V. Blobel, ${ }^{38}$ S. Bobrovskyi,${ }^{38}$ J. Draeger, ${ }^{38}$ H. Enderle, ${ }^{38}$ J. Erfle, ${ }^{38}$ U. Gebbert, ${ }^{38}$ M. Görner, ${ }^{38}$ T. Hermanns, ${ }^{38}$ R. S. Höing, ${ }^{38}$ K. Kaschube, ${ }^{38}$ G. Kaussen, ${ }^{38}$ H. Kirschenmann, ${ }^{38}$ R. Klanner, ${ }^{38}$ J. Lange, ${ }^{38}$ B. Mura ${ }^{38}$ F. Nowak, ${ }^{38}$ N. Pietsch, ${ }^{38}$ D. Rathjens,${ }^{38}$ C. Sander,${ }^{38}$ H. Schettler, ${ }^{38}$ P. Schleper, ${ }^{38}$ E. Schlieckau, ${ }^{38}$ A. Schmidt, ${ }^{38}$ M. Schröder, ${ }^{38}$

T. Schum,${ }^{38}$ M. Seidel,${ }^{38}$ H. Stadie, ${ }^{38}$ G. Steinbrück, ${ }^{38}$ J. Thomsen, ${ }^{38}$ C. Barth, ${ }^{39}$ J. Berger, ${ }^{39}$ T. Chwalek,${ }^{39}$ W. De Boer, ${ }^{39}$ A. Dierlamm, ${ }^{39}$ M. Feindt, ${ }^{39}$ M. Guthoff, ${ }^{39, b}$ C. Hackstein, ${ }^{39}$ F. Hartmann, ${ }^{39}$ M. Heinrich, ${ }^{39}$ H. Held, ${ }^{39}$ K. H. Hoffmann, ${ }^{39}$ S. Honc, ${ }^{39}$ U. Husemann, ${ }^{39}$ I. Katkov, ${ }^{39, m}$ J. R. Komaragiri, ${ }^{39}$ D. Martschei, ${ }^{39}$ S. Mueller, ${ }^{39}$ 
Th. Müller, ${ }^{39}$ M. Niegel,,${ }^{39}$ A. Nürnberg, ${ }^{39}$ O. Oberst,${ }^{39}$ A. Oehler, ${ }^{39}$ J. Ott, ${ }^{39}$ T. Peiffer,${ }^{39}$ G. Quast,,${ }^{39}$ K. Rabbertz, ${ }^{39}$ F. Ratnikov, ${ }^{39}$ N. Ratnikova, ${ }^{39}$ S. Röcker,${ }^{39}$ C. Saout,${ }^{39}$ A. Scheurer, ${ }^{39}$ F.-P. Schilling, ${ }^{39}$ M. Schmanau, ${ }^{39}$ G. Schott, ${ }^{39}$ H. J. Simonis, ${ }^{39}$ F. M. Stober, ${ }^{39}$ D. Troendle, ${ }^{39}$ R. Ulrich,,${ }^{39}$ J. Wagner-Kuhr,,${ }^{39}$ T. Weiler, ${ }^{39}$ M. Zeise, ${ }^{39}$

E. B. Ziebarth, ${ }^{39}$ G. Daskalakis, ${ }^{40}$ T. Geralis, ${ }^{40}$ S. Kesisoglou, ${ }^{40}$ A. Kyriakis, ${ }^{40}$ D. Loukas, ${ }^{40}$ I. Manolakos, ${ }^{40}$ A. Markou, ${ }^{40}$ C. Markou, ${ }^{40}$ C. Mavrommatis, ${ }^{40}$ E. Ntomari, ${ }^{40}$ L. Gouskos,${ }^{41}$ T. J. Mertzimekis, ${ }^{41}$ A. Panagiotou, ${ }^{41}$ N. Saoulidou, ${ }^{41}$ I. Evangelou, ${ }^{42}$ C. Foudas, ${ }^{42, b}$ P. Kokkas, ${ }^{42}$ N. Manthos, ${ }^{42}$ I. Papadopoulos, ${ }^{42}$ V. Patras, ${ }^{42}$ G. Bencze, ${ }^{43}$ C. Hajdu, ${ }^{43, b}$ P. Hidas, ${ }^{43}$ D. Horvath, ${ }^{43, o}$ K. Krajczar, ${ }^{43, p}$ B. Radics,${ }^{43}$ F. Sikler, ${ }^{43, b}$ V. Veszpremi, ${ }^{43}$ G. Vesztergombi ${ }^{43, p}$ N. Beni ${ }^{44}$ S. Czellar, ${ }^{44}$ J. Molnar, ${ }^{44}$ J. Palinkas, ${ }^{44}$ Z. Szillasi, ${ }^{44}$ J. Karancsi, ${ }^{45}$ P. Raics ${ }^{45}$ Z. L. Trocsanyi, ${ }^{45}$ B. Ujvari, ${ }^{45}$ S. B. Beri, ${ }^{46}$ V. Bhatnagar, ${ }^{46}$ N. Dhingra, ${ }^{46}$ R. Gupta, ${ }^{46}$ M. Jindal,${ }^{46}$ M. Kaur ${ }^{46}$ J. M. Kohli, ${ }^{46}$ M. Z. Mehta, ${ }^{46}$ N. Nishu, ${ }^{46}$ L. K. Saini,${ }^{46}$ A. Sharma, ${ }^{46}$ J. Singh ${ }^{46}$ S. P. Singh ${ }^{46}$ S. Ahuja ${ }^{47}$ B. C. Choudhary, ${ }^{47}$ A. Kumar, ${ }^{47}$ A. Kumar, ${ }^{47}$ S. Malhotra, ${ }^{47}$ M. Naimuddin, ${ }^{47}$ K. Ranjan,${ }^{47}$ V. Sharma, ${ }^{47}$ R. K. Shivpuri ${ }^{47}$ S. Banerjee, ${ }^{48}$ S. Bhattacharya ${ }^{48}$ S. Dutta, ${ }^{48}$ B. Gomber, ${ }^{48}$ Sa. Jain, ${ }^{48}$ Sh. Jain, ${ }^{48}$ R. Khurana ${ }^{48}$ S. Sarkar, ${ }^{48}$ A. Abdulsalam, ${ }^{49}$ R. K. Choudhury, ${ }^{49}$ D. Dutta, ${ }^{49}$ S. Kailas, ${ }^{49}$ V. Kumar, ${ }^{49}$ A. K. Mohanty, ${ }^{49, b}$ L. M. Pant,${ }^{49}$ P. Shukla, ${ }^{49}$ T. Aziz,${ }^{50}$ S. Ganguly, ${ }^{50}$ M. Guchait,${ }^{50, \mathrm{q}}$ A. Gurtu, ${ }^{50}$ M. Maity, ${ }^{50, \mathrm{r}}$ G. Majumder ${ }^{50}$ K. Mazumdar, ${ }^{50}$ G. B. Mohanty, ${ }^{50}$ B. Parida,${ }^{50}$ K. Sudhakar, ${ }^{50}$ N. Wickramage,${ }^{50}$ S. Banerjee,${ }^{51}$ S. Dugad, ${ }^{51}$ H. Arfaei, ${ }^{52}$ H. Bakhshiansohi, ${ }^{52, \mathrm{~s}}$ S. M. Etesami, ${ }^{52, \mathrm{t}}$ A. Fahim,${ }^{52, \mathrm{~s}}$ M. Hashemi, ${ }^{52}$ H. Hesari, ${ }^{52}$ A. Jafari, ${ }^{52, \mathrm{~s}}$ M. Khakzad, ${ }^{52}$ A. Mohammadi,${ }^{52, u}$ M. Mohammadi Najafabadi, ${ }^{52}$ S. Paktinat Mehdiabadi, ${ }^{52}$ B. Safarzadeh, ${ }^{52, v}$ M. Zeinali, ${ }^{52, t}$ M. Abbrescia, ${ }^{53 a, 53 b}$ L. Barbone, ${ }^{53 a, 53 b}$ C. Calabria, ${ }^{53 a, 53 b, b}$ S. S. Chhibra, ${ }^{53 a, 53 b}$ A. Colaleo, ${ }^{53 a}$ D. Creanza, ${ }^{53 a, 53 c}$ N. De Filippis, ${ }^{53 a, 53 c, b}$ M. De Palma, ${ }^{53 a, 53 b}$ L. Fiore, ${ }^{53 a}$ G. Iaselli, ${ }^{53 a, 53 c}$ L. Lusito, ${ }^{53 a, 53 b}$ G. Maggi, ${ }^{53 a, 53 c}$ M. Maggi, ${ }^{53 a}$ B. Marangelli, ${ }^{53 a, 53 b}$ S. My, ${ }^{53,53 c}$ S. Nuzzo, ${ }^{53 a, 53 b}$ N. Pacifico, ${ }^{53 a, 53 b}$ A. Pompili, ${ }^{53 a, 53 b}$ G. Pugliese, ${ }^{53 a, 53 c}$ G. Selvaggi, ${ }^{53 a, 53 b}$ L. Silvestris, ${ }^{53 a}$ G. Singh, ${ }^{53 a, 53 b}$ G. Zito, ${ }^{53 a}$ G. Abbiendi, ${ }^{54 a}$ A. C. Benvenuti, ${ }^{54 a}$

D. Bonacorsi, ${ }^{54 a, 54 b}$ S. Braibant-Giacomelli, ${ }^{54 a, 54 b}$ L. Brigliadori, ${ }^{54 a, 54 b}$ P. Capiluppi, ${ }^{54 a, 54 b}$ A. Castro, ${ }^{54 a, 54 b}$ F. R. Cavallo, ${ }^{54 a}$ M. Cuffiani, ${ }^{54 a, 54 b}$ G. M. Dallavalle, ${ }^{54 a}$ F. Fabbri, ${ }^{54 a}$ A. Fanfani, ${ }^{54 a, 54 b}$ D. Fasanella, ${ }^{54 a, 54 b, b}$ P. Giacomelli, ${ }^{54 a}$ C. Grandi, ${ }^{54 a}$ L. Guiducci, ${ }^{54 a}$ S. Marcellini, ${ }^{54 a}$ G. Masetti, ${ }^{54 a}$ M. Meneghelli, ${ }^{54 a, 54 b, b}$ A. Montanari, ${ }^{54 \mathrm{a}}$ F. L. Navarria, ${ }^{54 a, 54 b}$ F. Odorici, ${ }^{54 \mathrm{a}}$ A. Perrotta, ${ }^{54 \mathrm{a}}$ F. Primavera,${ }^{54,54 b}$ A. M. Rossi, ${ }^{54 a, 54 b}$ T. Rovelli, ${ }^{54 a, 54 b}$ G. Siroli, ${ }^{54 a, 54 b}$ R. Travaglini, ${ }^{54 a, 54 b}$ S. Albergo, ${ }^{55 a, 55 b}$ G. Cappello, ${ }^{55 a, 55 b}$ M. Chiorboli, ${ }^{55 a, 55 b}$ S. Costa, ${ }^{55 a, 55 b}$ R. Potenza, ${ }^{55 a, 55 b}$ A. Tricomi, ${ }^{55 a, 55 b}$ C. Tuve, ${ }^{55 a, 55 b}$ G. Barbagli, ${ }^{56 a}$ V. Ciulli, ${ }^{56 a, 56 b}$ C. Civinini, ${ }^{56 a}$ R. D' Alessandro, ${ }^{56 a, 56 b}$ E. Focardi, ${ }^{56 a, 56 b}$ S. Frosali, ${ }^{56 a, 56 b}$ E. Gallo, ${ }^{56 a}$ S. Gonzi, ${ }^{56 a, 56 b}$ M. Meschini, ${ }^{56 a}$ S. Paoletti, ${ }^{56 a}$ G. Sguazzoni, ${ }^{56 \mathrm{a}}$ A. Tropiano, ${ }^{56 \mathrm{a}, \mathrm{b}}$ L. Benussi, ${ }^{57} \mathrm{~S}$. Bianco, ${ }^{57}$ S. Colafranceschi,${ }^{57, \mathrm{w}}$ F. Fabbri, ${ }^{57}$ D. Piccolo, ${ }^{57}$ P. Fabbricatore, ${ }^{58}$ R. Musenich, ${ }^{58}$ A. Benaglia, ${ }^{59 a, 59 b, b}$ F. De Guio, ${ }^{59 a, 59 b}$ L. Di Matteo, ${ }^{59 a, 59 b, b}$ S. Fiorendi, ${ }^{59 a, 59 b}$ S. Gennai, ${ }^{59 a, b}$ A. Ghezzi, ${ }^{59 a, 59 b}$ S. Malvezzi, ${ }^{59 a}$ R. A. Manzoni, ${ }^{59 a, 59 b}$ A. Martelli, ${ }^{59 a, 59 b}$ A. Massironi, ${ }^{59 a, 59 b, b}$ D. Menasce, ${ }^{59 a}$ L. Moroni, ${ }^{59 a}$ M. Paganoni, ${ }^{59 a, 59 b}$ D. Pedrini, ${ }^{59 a}$ S. Ragazzi,${ }^{59 a, 59 b}$ N. Redaelli, ${ }^{59 a}$ S. Sala, ${ }^{59 a}$ T. Tabarelli de Fatis, ${ }^{59 a, 59 b}$ S. Buontempo, ${ }^{60 a}$ C. A. Carrillo Montoya,${ }^{60 a, b}$ N. Cavallo, ${ }^{60 a, x}$ A. De Cosa, ${ }^{60,60 b}$ O. Dogangun, ${ }^{60 a, 60 b}$ F. Fabozzi, ${ }^{60 a, x}$ A. O. M. Iorio, ${ }^{6 a, b}$ L. Lista, ${ }^{60 a}$ S. Meola, ${ }^{60 a, y}$ M. Merola, ${ }^{60 a, 60 b}$ P. Paolucci, ${ }^{60 a}$ P. Azzi, ${ }^{61 \mathrm{a}}$ N. Bacchetta, ${ }^{61 \mathrm{a}, \mathrm{b}}$ D. Bisello, ${ }^{61 \mathrm{a}, 61 \mathrm{~b}}$ A. Branca, ${ }^{61 \mathrm{a}, \mathrm{b}}$ R. Carlin, ${ }^{61 \mathrm{a}, 61 \mathrm{~b}}$ P. Checchia, ${ }^{61 \mathrm{a}}$ T. Dorigo, ${ }^{61 \mathrm{a}}$

U. Dosselli, ${ }^{61 \mathrm{a}}$ F. Gasparini, ${ }^{61 \mathrm{a}, 61 \mathrm{~b}}$ A. Gozzelino, ${ }^{61 \mathrm{a}}$ K. Kanishchev, ${ }^{61 \mathrm{a}, 61 \mathrm{c}}$ S. Lacaprara, ${ }^{61 \mathrm{a}}$ I. Lazzizzera, ${ }^{61 \mathrm{a}, 61 \mathrm{c}}$

M. Margoni, ${ }^{61 \mathrm{a}, 61 \mathrm{~b}}$ A. T. Meneguzzo, ${ }^{61 \mathrm{a}, 61 \mathrm{~b}}$ M. Nespolo, ${ }^{61 \mathrm{a}, \mathrm{b}}$ L. Perrozzi, ${ }^{61 \mathrm{a}} \mathrm{N}$. Pozzobon, ${ }^{61 \mathrm{a}, 61 \mathrm{~b}}$ P. Ronchese,${ }^{61 \mathrm{a}, 61 \mathrm{~b}}$ F. Simonetto, ${ }^{61 \mathrm{a}, 61 \mathrm{~b}}$ E. Torassa, ${ }^{61 \mathrm{a}}$ M. Tosi, ${ }^{61 \mathrm{a}, 61 \mathrm{~b}, \mathrm{~b}}$ S. Vanini, ${ }^{61 \mathrm{a}, 61 \mathrm{~b}}$ P. Zotto, ${ }^{61 \mathrm{a}, 61 \mathrm{~b}}$ G. Zumerle, ${ }^{61 \mathrm{a}, 61 \mathrm{~b}}$

M. Gabusi, ${ }^{62 a, 62 b}$ S. P. Ratti, ${ }^{62 a, 62 b}$ C. Riccardi, ${ }^{62 a, 62 b}$ P. Torre, ${ }^{62 a, 62 b}$ P. Vitulo, ${ }^{62 a, 62 b}$ G. M. Bilei, ${ }^{63 a}$ L. Fanò,${ }^{63 a, 63 b}$ P. Lariccia, ${ }^{63 a, 63 b}$ A. Lucaroni, ${ }^{63 a, 63 b, b}$ G. Mantovani, ${ }^{63 a, 63 b}$ M. Menichelli, ${ }^{63 a}$ A. Nappi, ${ }^{63 a, 63 b}$ F. Romeo, ${ }^{63 a, 63 b}$ A. Saha, ${ }^{63 a}$ A. Santocchia, ${ }^{63 a, 63 b}$ S. Taroni, ${ }^{63 a, 63 b, b}$ P. Azzurri, ${ }^{64 a, 64 c}$ G. Bagliesi, ${ }^{64 a}$ T. Boccali, ${ }^{64 a}$ G. Broccolo, ${ }^{64 a, 64 c}$ R. Castaldi, ${ }^{64 a}$ R. T. D’Agnolo, ${ }^{64 a, 64 c}$ R. Dell'Orso, ${ }^{64 a}$ F. Fiori, ${ }^{64 a, 64 b, b}$ L. Foà, ${ }^{64 a, 64 c}$ A. Giassi, ${ }^{64 a}$ A. Kraan, ${ }^{64 a}$ F. Ligabue, ${ }^{64 a, 64 c}$ T. Lomtadze, ${ }^{64 a}$ L. Martini, ${ }^{64 a, z}$ A. Messineo, ${ }^{64 a, 64 b}$ F. Palla, ${ }^{64 a}$ F. Palmonari, ${ }^{64 a}$ A. Rizzi, ${ }^{64 a, 64 b}$ A. T. Serban, ${ }^{64 a, a a}$ P. Spagnolo, ${ }^{64 a}$ P. Squillacioti, ${ }^{64 a, b}$ R. Tenchini, ${ }^{64 a}$ G. Tonelli, ${ }^{64 a, 64 b, b}$ A. Venturi, ${ }^{64 a, b}$ P. G. Verdini, ${ }^{64 a}$ L. Barone,${ }^{65 a, 65 b}$ F. Cavallari, ${ }^{65 a}$ D. Del Re,${ }^{65 a, 65 b, b}$ M. Diemoz, ${ }^{65 a}$ C. Fanelli, ${ }^{65 a, 65 b}$ M. Grassi ${ }^{65 a, b}$ E. Longo, ${ }^{65 a, 65 b}$ P. Meridiani, ${ }^{65 a, b}$ F. Micheli, ${ }^{65 a, 65 b}$ S. Nourbakhsh, ${ }^{65 a}$ G. Organtini, ${ }^{65 a, 65 b}$ F. Pandolfi, ${ }^{65 a, 65 b}$

R. Paramatti, ${ }^{65 a}$ S. Rahatlou, ${ }^{65 a, 65 b}$ M. Sigamani, ${ }^{65 a}$ L. Soffi, ${ }^{65 a, 65 b}$ N. Amapane, ${ }^{66 a, 66 b}$ R. Arcidiacono, ${ }^{66 a, 66 c}$ S. Argiro, ${ }^{66 a, 66 b}$ M. Arneodo, ${ }^{66 a, 66 c}$ C. Biino, ${ }^{66 a}$ C. Botta, ${ }^{66 a, 66 b}$ N. Cartiglia, ${ }^{66 a}$ R. Castello, ${ }^{66 a, 66 b}$ M. Costa, ${ }^{66 a, 66 b}$ N. Demaria ${ }^{66 \mathrm{a}}$ A. Graziano, ${ }^{66 \mathrm{a}, 66 \mathrm{~b}}$ C. Mariotti, ${ }^{66 \mathrm{a}, \mathrm{b}}$ S. Maselli, ${ }^{66 \mathrm{a}}$ E. Migliore ${ }^{66 \mathrm{a}, 66 \mathrm{~b}}$ V. Monaco, ${ }^{66 \mathrm{a}, 66 \mathrm{~b}}$ M. Musich, ${ }^{66 a, b}$ M. M. Obertino, ${ }^{66 a, 66 c}$ N. Pastrone, ${ }^{66 \mathrm{a}}$ M. Pelliccioni, ${ }^{66 \mathrm{a}}$ A. Potenza, ${ }^{66,66 \mathrm{~b}}$ A. Romero, ${ }^{66 \mathrm{a}, 66 \mathrm{~b}}$ M. Ruspa,${ }^{66,66 c}$ R. Sacchi, ${ }^{66 a, 66 b}$ V. Sola,${ }^{66 a, 66 b}$ A. Solano, ${ }^{66 a, 66 b}$ A. Staiano, ${ }^{66 a}$ A. Vilela Pereira, ${ }^{66 a}$ S. Belforte,${ }^{67 a}$ 
F. Cossutti, ${ }^{67 a}$ G. Della Ricca, ${ }^{67 a, 67 b}$ B. Gobbo, ${ }^{67 a}$ M. Marone, ${ }^{67 a, 67 b, b}$ D. Montanino, ${ }^{67 a, 67 b, b}$ A. Penzo, ${ }^{67 a}$

A. Schizzi, ${ }^{67 a, 67 b}$ S. G. Heo, ${ }^{68}$ T. Y. Kim, ${ }^{68}$ S. K. Nam, ${ }^{68}$ S. Chang, ${ }^{69}$ J. Chung, ${ }^{69}$ D. H. Kim, ${ }^{69}$ G. N. Kim, ${ }^{69}$ D. J. Kong, ${ }^{69}$ H. Park, ${ }^{69}$ S. R. Ro, ${ }^{69}$ D. C. Son, ${ }^{69}$ T. Son, ${ }^{69}$ J. Y. Kim, ${ }^{70}$ Zero J. Kim, ${ }^{70}$ S. Song, ${ }^{70}$ H. Y. Jo, ${ }^{71}$ S. Choi, ${ }^{72}$ D. Gyun, ${ }^{72}$ B. Hong, ${ }^{72}$ M. Jo, ${ }^{72}$ H. Kim, ${ }^{72}$ T. J. Kim, ${ }^{72}$ K. S. Lee, ${ }^{72}$ D. H. Moon, ${ }^{72}$ S. K. Park, ${ }^{72}$ E. Seo, ${ }^{72}$ M. Choi, ${ }^{73}$ S. Kang, ${ }^{73}$ H. Kim, ${ }^{73}$ J. H. Kim,${ }^{73}$ C. Park, ${ }^{73}$ I. C. Park, ${ }^{73}$ S. Park, ${ }^{73}$ G. Ryu, ${ }^{73}$ Y. Cho, ${ }^{74}$ Y. Choi, ${ }^{74}$ Y. K. Choi, ${ }^{74}$ J. Goh, ${ }^{74}$ M. S. Kim, ${ }^{74}$ E. Kwon, ${ }^{74}$ B. Lee, ${ }^{74}$ J. Lee, ${ }^{74}$ S. Lee, ${ }^{74}$ H. Seo, ${ }^{74}$ I. Yu, ${ }^{74}$ M. J. Bilinskas, ${ }^{75}$ I. Grigelionis, $^{75}$ M. Janulis, ${ }^{75}$ A. Juodagalvis, ${ }^{75}$ H. Castilla-Valdez, ${ }^{76}$ E. De La Cruz-Burelo, ${ }^{76}$ I. Heredia-de La Cruz, ${ }^{76}$ R. Lopez-Fernandez, ${ }^{76}$ R. Magaña Villalba, ${ }^{76} \mathrm{~J}_{\text {. Martínez-Ortega, }}{ }^{76}$ A. Sánchez-Hernández, ${ }^{76}$

L. M. Villasenor-Cendejas, ${ }^{76}$ S. Carrillo Moreno, ${ }^{77}$ F. Vazquez Valencia, ${ }^{77}$ H. A. Salazar Ibarguen, ${ }^{78}$

E. Casimiro Linares, ${ }^{79}$ A. Morelos Pineda, ${ }^{79}$ M. A. Reyes-Santos, ${ }^{79}$ D. Krofcheck, ${ }^{80}$ A. J. Bell, ${ }^{81}$ P. H. Butler, ${ }^{81}$

R. Doesburg, ${ }^{81}$ S. Reucroft, ${ }^{81}$ H. Silverwood, ${ }^{81}$ M. Ahmad, ${ }^{82}$ M. I. Asghar, ${ }^{82}$ H. R. Hoorani, ${ }^{82}$ S. Khalid, ${ }^{82}$ W. A. Khan, ${ }^{82}$ T. Khurshid, ${ }^{82}$ S. Qazi, ${ }^{82}$ M. A. Shah, ${ }^{82}$ M. Shoaib, ${ }^{82}$ G. Brona, ${ }^{83}$ K. Bunkowski, ${ }^{83}$ M. Cwiok, ${ }^{83}$ W. Dominik, ${ }^{83}$ K. Doroba, ${ }^{83}$ A. Kalinowski, ${ }^{83}$ M. Konecki, ${ }^{83}$ J. Krolikowski, ${ }^{83}$ H. Bialkowska, ${ }^{84}$ B. Boimska, ${ }^{84}$ T. Frueboes, ${ }^{84}$ R. Gokieli, ${ }^{84}$ M. Górski, ${ }^{84}$ M. Kazana, ${ }^{84}$ K. Nawrocki, ${ }^{84}$ K. Romanowska-Rybinska, ${ }^{84}$ M. Szleper, ${ }^{84}$ G. Wrochna, ${ }^{84}$ P. Zalewski, ${ }^{84}$ N. Almeida, ${ }^{85}$ P. Bargassa, ${ }^{85}$ A. David, ${ }^{85}$ P. Faccioli, ${ }^{85}$ P. G. Ferreira Parracho, ${ }^{85}$ M. Gallinaro, ${ }^{85}$ P. Musella, ${ }^{85}$ J. Seixas, ${ }^{85}$ J. Varela, ${ }^{85}$ P. Vischia, ${ }^{85}$ S. Afanasiev, ${ }^{86}$ I. Belotelov, ${ }^{86}$ P. Bunin, ${ }^{86}$ M. Gavrilenko, ${ }^{86}$ I. Golutvin, ${ }^{86}$ A. Kamenev, ${ }^{86}$ V. Karjavin, ${ }^{86}$ G. Kozlov, ${ }^{86}$ A. Lanev, ${ }^{86}$ A. Malakhov, ${ }^{86}$ P. Moisenz, ${ }^{86}$

V. Palichik, ${ }^{86}$ V. Perelygin, ${ }^{86}$ S. Shmatov, ${ }^{86}$ V. Smirnov, ${ }^{86}$ A. Volodko, ${ }^{86}$ A. Zarubin, ${ }^{86}$ S. Evstyukhin, ${ }^{87}$

V. Golovtsov, ${ }^{87}$ Y. Ivanov, ${ }^{87}$ V. Kim, ${ }^{87}$ P. Levchenko, ${ }^{87}$ V. Murzin, ${ }^{87}$ V. Oreshkin, ${ }^{87}$ I. Smirnov, ${ }^{87}$ V. Sulimov, ${ }^{87}$

L. Uvarov, ${ }^{87}$ S. Vavilov, ${ }^{87}$ A. Vorobyev, ${ }^{87}$ An. Vorobyev, ${ }^{87}$ Yu. Andreev, ${ }^{88}$ A. Dermenev, ${ }^{88}$ S. Gninenko, ${ }^{88}$

N. Golubev, ${ }^{88}$ M. Kirsanov, ${ }^{88}$ N. Krasnikov, ${ }^{88}$ V. Matveev, ${ }^{88}$ A. Pashenkov, ${ }^{88}$ D. Tlisov, ${ }^{88}$ A. Toropin, ${ }^{88}$ V. Epshteyn, ${ }^{89}$ M. Erofeeva, ${ }^{89}$ V. Gavrilov, ${ }^{89}$ M. Kossov, ${ }^{89, b}$ N. Lychkovskaya, ${ }^{89}$ V. Popov, ${ }^{89}$ G. Safronov, ${ }^{89}$ S. Semenov, ${ }^{89}$ V. Stolin, ${ }^{89}$ E. Vlasov, ${ }^{89}$ A. Zhokin, ${ }^{89}$ A. Belyaev, ${ }^{90}$ E. Boos, ${ }^{90}$ A. Ershov, ${ }^{90}$ A. Gribushin, ${ }^{90}$ V. Klyukhin, ${ }^{90}$ O. Kodolova, ${ }^{90}$ V. Korotkikh, ${ }^{90}$ I. Lokhtin, ${ }^{90}$ A. Markina, ${ }^{90}$ S. Obraztsov, ${ }^{90}$ M. Perfilov, ${ }^{90}$ S. Petrushanko, ${ }^{90}$ L. Sarycheva, ${ }^{90, a}$ V. Savrin, ${ }^{90}$ A. Snigirev, ${ }^{90}$ I. Vardanyan, ${ }^{90}$ V. Andreev, ${ }^{91}$ M. Azarkin, ${ }^{91}$ I. Dremin, ${ }^{91}$ M. Kirakosyan, ${ }^{91}$ A. Leonidov, ${ }^{91}$ G. Mesyats, ${ }^{91}$ S. V. Rusakov, ${ }^{91}$ A. Vinogradov, ${ }^{91}$ I. Azhgirey, ${ }^{92}$ I. Bayshev, ${ }^{92}$ S. Bitioukov, ${ }^{92}$ V. Grishin, ${ }^{92, b}$ V. Kachanov, ${ }^{92}$ D. Konstantinov, ${ }^{92}$ A. Korablev, ${ }^{92}$ V. Krychkine, ${ }^{92}$ V. Petrov, ${ }^{92}$ R. Ryutin, ${ }^{92}$ A. Sobol, ${ }^{92}$ L. Tourtchanovitch, ${ }^{92}$ S. Troshin, ${ }^{92}$ N. Tyurin, ${ }^{92}$ A. Uzunian, ${ }^{92}$ A. Volkov, ${ }^{92}$ P. Adzic, ${ }^{93, b b}$ M. Djordjevic, ${ }^{93}$ M. Ekmedzic, ${ }^{93}$ D. Krpic, ${ }^{93, b b}$ J. Milosevic, ${ }^{93}$ M. Aguilar-Benitez, ${ }^{94}$

J. Alcaraz Maestre, ${ }^{94}$ P. Arce, ${ }^{94}$ C. Battilana, ${ }^{94}$ E. Calvo, ${ }^{94}$ M. Cerrada, ${ }^{94}$ M. Chamizo Llatas, ${ }^{94}$ N. Colino, ${ }^{94}$ B. De La Cruz, ${ }^{94}$ A. Delgado Peris, ${ }^{94}$ C. Diez Pardos, ${ }^{94}$ D. Domínguez Vázquez, ${ }^{94}$ C. Fernandez Bedoya, ${ }^{94}$ J. P. Fernández Ramos, ${ }^{94}$ A. Ferrando, ${ }^{94}$ J. Flix, ${ }^{94}$ M. C. Fouz,${ }^{94}$ P. Garcia-Abia, ${ }^{94}$ O. Gonzalez Lopez, ${ }^{94}$ S. Goy Lopez, ${ }^{94}$ J. M. Hernandez, ${ }^{94}$ M. I. Josa, ${ }^{94}$ G. Merino, ${ }^{94}$ J. Puerta Pelayo, ${ }^{94}$ I. Redondo, ${ }^{94}$ L. Romero, ${ }^{94}$ J. Santaolalla, ${ }^{94}$ M. S. Soares, ${ }^{94}$ C. Willmott, ${ }^{94}$ C. Albajar, ${ }^{95}$ G. Codispoti, ${ }^{95}$ J. F. de Trocóniz, ${ }^{95}$ J. Cuevas, ${ }^{96}$ J. Fernandez Menendez, ${ }^{96}$ S. Folgueras, ${ }^{96}$ I. Gonzalez Caballero, ${ }^{96}$ L. Lloret Iglesias, ${ }^{96}$ J. Piedra Gomez, ${ }^{96, c c}$ J. M. Vizan Garcia, ${ }^{96}$ J. A. Brochero Cifuentes, ${ }^{97}$ I. J. Cabrillo, ${ }^{97}$ A. Calderon, ${ }^{97}$ S. H. Chuang, ${ }^{97}$

J. Duarte Campderros, ${ }^{97}$ M. Felcini, ${ }^{97, d d}$ M. Fernandez, ${ }^{97}$ G. Gomez, ${ }^{97}$ J. Gonzalez Sanchez, ${ }^{97}$ C. Jorda, ${ }^{97}$ P. Lobelle Pardo, ${ }^{97}$ A. Lopez Virto, ${ }^{97}$ J. Marco, ${ }^{97}$ R. Marco, ${ }^{97}$ C. Martinez Rivero, ${ }^{97}$ F. Matorras, ${ }^{97}$

F. J. Munoz Sanchez, ${ }^{97}$ T. Rodrigo, ${ }^{97}$ A. Y. Rodríguez-Marrero, ${ }^{97}$ A. Ruiz-Jimeno, ${ }^{97}$ L. Scodellaro, ${ }^{97}$ M. Sobron Sanudo, ${ }^{97}$ I. Vila, ${ }^{97}$ R. Vilar Cortabitarte, ${ }^{97}$ D. Abbaneo, ${ }^{98}$ E. Auffray, ${ }^{98}$ G. Auzinger, ${ }^{98}$ P. Baillon, ${ }^{98}$ A. H. Ball, ${ }^{98}$ D. Barney, ${ }^{98}$ C. Bernet, ${ }^{98, f}$ G. Bianchi, ${ }^{98}$ P. Bloch, ${ }^{98}$ A. Bocci, ${ }^{98}$ A. Bonato, ${ }^{98}$ H. Breuker, ${ }^{98}$ T. Camporesi, ${ }^{98}$ G. Cerminara, ${ }^{98}$ T. Christiansen, ${ }^{98}$ J. A. Coarasa Perez, ${ }^{98}$ D. D'Enterria, ${ }^{98}$ A. De Roeck, ${ }^{98}$ S. Di Guida, ${ }^{98}$ M. Dobson, ${ }^{98}$ N. Dupont-Sagorin, ${ }^{98}$ A. Elliott-Peisert, ${ }^{98}$ B. Frisch, ${ }^{98}$ W. Funk, ${ }^{98}$ G. Georgiou, ${ }^{98}$ M. Giffels, ${ }^{98}$ D. Gigi, ${ }^{98}$ K. Gill, ${ }^{98}$ D. Giordano, ${ }^{98}$ M. Giunta, ${ }^{98}$ F. Glege, ${ }^{98}$ R. Gomez-Reino Garrido, ${ }^{98}$ P. Govoni, ${ }^{98}$

S. Gowdy, ${ }^{98}$ R. Guida, ${ }^{98}$ M. Hansen, ${ }^{98}$ P. Harris, ${ }^{98}$ C. Hartl, ${ }^{98}$ J. Harvey, ${ }^{98}$ B. Hegner, ${ }^{98}$ A. Hinzmann, ${ }^{98}$ V. Innocente, ${ }^{98}$ P. Janot,${ }^{98}$ K. Kaadze,${ }^{98}$ E. Karavakis, ${ }^{98}$ K. Kousouris, ${ }^{98}$ P. Lecoq, ${ }^{98}$ P. Lenzi, ${ }^{98}$ C. Lourenço, ${ }^{98}$ T. Mäki, ${ }^{98}$ M. Malberti, ${ }^{98}$ L. Malgeri, ${ }^{98}$ M. Mannelli, ${ }^{98}$ L. Masetti, ${ }^{98}$ F. Meijers, ${ }^{98}$ S. Mersi, ${ }^{98}$ E. Meschi, ${ }^{98}$

R. Moser, ${ }^{98}$ M. U. Mozer, ${ }^{98}$ M. Mulders, ${ }^{98}$ E. Nesvold, ${ }^{98}$ M. Nguyen, ${ }^{98}$ T. Orimoto, ${ }^{98}$ L. Orsini, $^{98}$

E. Palencia Cortezon, ${ }^{98}$ E. Perez, ${ }^{98}$ A. Petrilli, ${ }^{98}$ A. Pfeiffer, ${ }^{98}$ M. Pierini, ${ }^{98}$ M. Pimiä, ${ }^{98}$ D. Piparo, ${ }^{98}$ G. Polese, ${ }^{98}$ L. Quertenmont, ${ }^{98}$ A. Racz, ${ }^{98}$ W. Reece, ${ }^{98}$ J. Rodrigues Antunes, ${ }^{98}$ G. Rolandi, ${ }^{98, e e}$ T. Rommerskirchen, ${ }^{98}$ C. Rovelli, ${ }^{98, f f}$ M. Rovere, ${ }^{98}$ H. Sakulin, ${ }^{98}$ F. Santanastasio, ${ }^{98}$ C. Schäfer, ${ }^{98}$ C. Schwick, ${ }^{98}$ I. Segoni, ${ }^{98}$ S. Sekmen, ${ }^{98}$ 
A. Sharma, ${ }^{98}$ P. Siegrist,${ }^{98}$ P. Silva,${ }^{98}$ M. Simon, ${ }^{98}$ P. Sphicas,${ }^{98, g g}$ D. Spiga,${ }^{98}$ M. Spiropulu, ${ }^{98, e}$ M. Stoye,${ }^{98}$ A. Tsirou, ${ }^{98}$ G. I. Veres, ${ }^{98, p}$ J. R. Vlimant,${ }^{98}$ H. K. Wöhri, ${ }^{98}$ S. D. Worm, $,{ }^{98, \text { hh W. D. Zeuner },{ }^{98} \text { W. Bertl, }{ }^{99} \text { K. Deiters }},{ }^{99}$ W. Erdmann, ${ }^{99}$ K. Gabathuler, ${ }^{99}$ R. Horisberger, ${ }^{99}$ Q. Ingram, ${ }^{99}$ H. C. Kaestli, ${ }^{99}$ S. König,${ }^{99}$ D. Kotlinski, ${ }^{99}$ U. Langenegger, ${ }^{99}$ F. Meier, ${ }^{99}$ D. Renker, ${ }^{99}$ T. Rohe, ${ }^{99}$ J. Sibille,,${ }^{99}$,i L. Bäni, ${ }^{100}$ P. Bortignon, ${ }^{100}$ M. A. Buchmann, ${ }^{100}$ B. Casal, ${ }^{100}$ N. Chanon, ${ }^{100}$ Z. Chen, ${ }^{100}$ A. Deisher, ${ }^{100}$ G. Dissertori, ${ }^{100}$ M. Dittmar, ${ }^{100}$ M. Dünser,${ }^{100}$ J. Eugster, ${ }^{100}$ K. Freudenreich, ${ }^{100}$ C. Grab, ${ }^{100}$ P. Lecomte, ${ }^{100}$ W. Lustermann, ${ }^{100}$ A. C. Marini, ${ }^{100}$ P. Martinez Ruiz del Arbol, ${ }^{100}$ N. Mohr ${ }^{100}$ F. Moortgat, ${ }^{100}$ C. Nägeli, ${ }^{100, j j}$ P. Nef, ${ }^{100}$ F. Nessi-Tedaldi, ${ }^{100}$ L. Pape,${ }^{100}$ F. Pauss, ${ }^{100}$ M. Peruzzi, ${ }^{100}$ F. J. Ronga, ${ }^{100}$ M. Rossini, ${ }^{100}$ L. Sala, ${ }^{100}$ A. K. Sanchez, ${ }^{100}$ A. Starodumov, ${ }^{100, k k}$ B. Stieger, ${ }^{100}$ M. Takahashi, ${ }^{100}$ L. Tauscher, ${ }^{100, a}$ A. Thea, ${ }^{100}$ K. Theofilatos, ${ }^{100}$ D. Treille, ${ }^{100}$ C. Urscheler, ${ }^{100}$ R. Wallny, ${ }^{100}$ H. A. Weber, ${ }^{100}$ L. Wehrli, ${ }^{100}$ E. Aguilo, ${ }^{101}$ C. Amsler,${ }^{101}$ V. Chiochia, ${ }^{101}$ S. De Visscher, ${ }^{101}$ C. Favaro, ${ }^{101}$ M. Ivova Rikova, ${ }^{101}$ B. Millan Mejias, ${ }^{101}$ P. Otiougova, ${ }^{101}$ P. Robmann, ${ }^{101}$ H. Snoek, ${ }^{101}$ S. Tupputi, ${ }^{101}$ M. Verzetti, ${ }^{101}$ Y. H. Chang, ${ }^{102}$ K. H. Chen, ${ }^{102}$ A. Go,${ }^{102}$ C. M. Kuo,${ }^{102}$ S. W. Li, ${ }^{102}$ W. Lin, ${ }^{102}$ Z. K. Liu,${ }^{102}$ Y. J. Lu, ${ }^{102}$ D. Mekterovic,${ }^{102}$ A. P. Singh, ${ }^{102}$ R. Volpe, ${ }^{102}$ S. S. Yu, ${ }^{102}$ P. Bartalini, ${ }^{103}$ P. Chang, ${ }^{103}$ Y. H. Chang, ${ }^{103}$ Y. W. Chang, ${ }^{103}$ Y. Chao, ${ }^{103}$ K. F. Chen, ${ }^{103}$ C. Dietz, ${ }^{103}$ U. Grundler, ${ }^{103}$ W.-S. Hou, ${ }^{103}$ Y. Hsiung, ${ }^{103}$ K. Y. Kao, ${ }^{103}$ Y. J. Lei, ${ }^{103}$ R.-S. Lu, ${ }^{103}$

D. Majumder, ${ }^{103}$ E. Petrakou, ${ }^{103}$ X. Shi, ${ }^{103}$ J. G. Shiu, ${ }^{103}$ Y. M. Tzeng, ${ }^{103}$ M. Wang, ${ }^{103}$ A. Adiguzel,,${ }^{104}$ M. N. Bakirci, ${ }^{104,11}$ S. Cerci, ${ }^{104, m m}$ C. Dozen, ${ }^{104}$ I. Dumanoglu, ${ }^{104}$ E. Eskut, ${ }^{104}$ S. Girgis, ${ }^{104}$ G. Gokbulut, ${ }^{104}$ I. Hos, ${ }^{104}$ E. E. Kangal, ${ }^{104}$ G. Karapinar, ${ }^{104}$ A. Kayis Topaksu, ${ }^{104}$ G. Onengut, ${ }^{104}$ K. Ozdemir, ${ }^{104}$ S. Ozturk, ${ }^{104, n n}$ A. Polatoz, ${ }^{104}$ K. Sogut, ${ }^{104, \text { oo }}$ D. Sunar Cerci, ${ }^{104, \mathrm{~mm}}$ B. Tali, ${ }^{104, \mathrm{~mm}}$ H. Topakli, ${ }^{104,11}$ L. N. Vergili, ${ }^{104}$ M. Vergili, ${ }^{104}$ I. V. Akin, ${ }^{105}$ T. Aliev, ${ }^{105}$ B. Bilin, ${ }^{105}$ S. Bilmis, ${ }^{105}$ M. Deniz, ${ }^{105}$ H. Gamsizkan, ${ }^{105}$ A. M. Guler, ${ }^{105}$ K. Ocalan, ${ }^{105}$ A. Ozpineci, ${ }^{105}$ M. Serin, ${ }^{105}$ R. Sever, ${ }^{105}$ U. E. Surat, ${ }^{105}$ M. Yalvac,,${ }^{105}$ E. Yildirim, ${ }^{105}$ M. Zeyrek, ${ }^{105}$ M. Deliomeroglu, ${ }^{106}$ E. Gülmez, ${ }^{106}$ B. Isildak, ${ }^{106}$ M. Kaya, ${ }^{106, p p}$ O. Kaya, ${ }^{106, p p}$ S. Ozkorucuklu, ${ }^{106, q q}$ N. Sonmez, ${ }^{106, \text { rr }}$ K. Cankocak, ${ }^{107}$ L. Levchuk, ${ }^{108}$ F. Bostock, ${ }^{109}$ J. J. Brooke, ${ }^{109}$ E. Clement, ${ }^{109}$ D. Cussans, ${ }^{109}$ H. Flacher, ${ }^{109}$ R. Frazier, ${ }^{109}$ J. Goldstein, ${ }^{109}$ M. Grimes, ${ }^{109}$ G. P. Heath,${ }^{109}$ H. F. Heath, ${ }^{109}$ L. Kreczko, ${ }^{109}$ S. Metson, ${ }^{109}$ D. M. Newbold, ${ }^{109, \text { hh }}$ K. Nirunpong, ${ }^{109}$ A. Poll, ${ }^{109}$ S. Senkin, ${ }^{109}$ V. J. Smith,${ }^{109}$ T. Williams, ${ }^{109}$ L. Basso, ${ }^{110, \text { ss }}$ A. Belyaev, ${ }^{110, \text { ss }}$ C. Brew, ${ }^{110}$ R. M. Brown, ${ }^{110}$ D. J. A. Cockerill, ${ }^{110}$ J. A. Coughlan, ${ }^{110}$ K. Harder, ${ }^{110}$ S. Harper, ${ }^{110}$ J. Jackson, ${ }^{110}$ B. W. Kennedy, ${ }^{110}$

E. Olaiya ${ }^{110}$ D. Petyt, ${ }^{110}$ B. C. Radburn-Smith, ${ }^{110}$ C. H. Shepherd-Themistocleous, ${ }^{110}$ I. R. Tomalin, ${ }^{10}$

W. J. Womersley, ${ }^{110}$ R. Bainbridge, ${ }^{111}$ G. Ball, ${ }^{111}$ R. Beuselinck, ${ }^{111}$ O. Buchmuller ${ }^{111}$ D. Colling, ${ }^{111}$ N. Cripps,${ }^{111}$ M. Cutajar, ${ }^{111}$ P. Dauncey, ${ }^{111}$ G. Davies,${ }^{111}$ M. Della Negra, ${ }^{111}$ W. Ferguson, ${ }^{111}$ J. Fulcher ${ }^{111}$ D. Futyan, ${ }^{111}$ A. Gilbert, ${ }^{111}$ A. Guneratne Bryer,${ }^{111}$ G. Hall,${ }^{111}$ Z. Hatherell, ${ }^{111}$ J. Hays, ${ }^{111}$ G. Iles, ${ }^{111}$ M. Jarvis,${ }^{111}$

G. Karapostoli, ${ }^{111}$ L. Lyons, ${ }^{111}$ A.-M. Magnan, ${ }^{111}$ J. Marrouche,${ }^{111}$ B. Mathias, ${ }^{111}$ R. Nandi, ${ }^{111}$ J. Nash, ${ }^{111}$ A. Nikitenko, ${ }^{111, \mathrm{kk}}$ A. Papageorgiou, ${ }^{111}$ J. Pela,${ }^{111, \mathrm{~b}}$ M. Pesaresi, ${ }^{111}$ K. Petridis,${ }^{111}$ M. Pioppi,${ }^{111, \mathrm{tt}}$

D. M. Raymond, ${ }^{111}$ S. Rogerson, ${ }^{111}$ N. Rompotis,${ }^{111}$ A. Rose,${ }^{111}$ M. J. Ryan, ${ }^{111}$ C. Seez, ${ }^{111}$ P. Sharp, ${ }^{111, a}$ A. Sparrow, ${ }^{111}$ A. Tapper, ${ }^{111}$ M. Vazquez Acosta, ${ }^{111}$ T. Virdee, ${ }^{111}$ S. Wakefield, ${ }^{111}$ N. Wardle, ${ }^{111}$ T. Whyntie,${ }^{111}$ M. Barrett, ${ }^{112}$ M. Chadwick, ${ }^{112}$ J. E. Cole, ${ }^{112}$ P. R. Hobson, ${ }^{112}$ A. Khan, ${ }^{112}$ P. Kyberd, ${ }^{112}$ D. Leggat,${ }^{112}$ D. Leslie, ${ }^{112}$ W. Martin, ${ }^{112}$ I. D. Reid, ${ }^{112}$ P. Symonds, ${ }^{112}$ L. Teodorescu, ${ }^{112}$ M. Turner, ${ }^{112}$ K. Hatakeyama, ${ }^{113}$ H. Liu,,${ }^{113}$

T. Scarborough, ${ }^{113}$ C. Henderson, ${ }^{114}$ P. Rumerio, ${ }^{114}$ A. Avetisyan,,${ }^{15}$ T. Bose, ${ }^{115}$ C. Fantasia, ${ }^{115}$ A. Heister, ${ }^{115}$ J. St. John, ${ }^{115}$ P. Lawson, ${ }^{115}$ D. Lazic,,${ }^{15}$ J. Rohlf, ${ }^{115}$ D. Sperka, ${ }^{115}$ L. Sulak,,${ }^{115}$ J. Alimena, ${ }^{116}$ S. Bhattacharya, ${ }^{116}$ D. Cutts, ${ }^{116}$ A. Ferapontov, ${ }^{116}$ U. Heintz, ${ }^{116}$ S. Jabeen, ${ }^{116}$ G. Kukartsev, ${ }^{116}$ G. Landsberg, ${ }^{116}$ M. Luk, ${ }^{116}$ M. Narain, ${ }^{116}$ D. Nguyen, ${ }^{116}$ M. Segala, ${ }^{116}$ T. Sinthuprasith, ${ }^{116}$ T. Speer, ${ }^{116}$ K. V. Tsang, ${ }^{116}$ R. Breedon, ${ }^{117}$ G. Breto, ${ }^{117}$ M. Calderon De La Barca Sanchez, ${ }^{117}$ S. Chauhan, ${ }^{117}$ M. Chertok,${ }^{117}$ J. Conway, ${ }^{117}$ R. Conway, ${ }^{117}$ P. T. Cox ${ }^{117}$ J. Dolen, ${ }^{117}$ R. Erbacher,${ }^{117}$ M. Gardner, ${ }^{117}$ R. Houtz, ${ }^{117}$ W. Ko, ${ }^{117}$ A. Kopecky, ${ }^{117}$ R. Lander, ${ }^{117}$ O. Mall, ${ }^{117}$ T. Miceli, ${ }^{117}$ R. Nelson, ${ }^{117}$ D. Pellett, ${ }^{117}$ B. Rutherford, ${ }^{117}$ M. Searle, ${ }^{117}$ J. Smith, ${ }^{117}$ M. Squires, ${ }^{117}$ M. Tripathi, ${ }^{117}$ R. Vasquez Sierra, ${ }^{117}$ V. Andreev, ${ }^{118}$ D. Cline,${ }^{118}$ R. Cousins, ${ }^{118}$ J. Duris ${ }^{118}$ S. Erhan, ${ }^{118}$ P. Everaerts, ${ }^{118}$ C. Farrell, ${ }^{118}$ J. Hauser, ${ }^{118}$ M. Ignatenko, ${ }^{118}$ C. Plager, ${ }^{118}$ G. Rakness, ${ }^{118}$ P. Schlein, ${ }^{18, a}$ J. Tucker, ${ }^{118}$ V. Valuev, ${ }^{118}$ M. Weber, ${ }^{118}$ J. Babb, ${ }^{119}$ R. Clare, ${ }^{119}$ M. E. Dinardo, ${ }^{119}$ J. Ellison, ${ }^{119}$ J. W. Gary, ${ }^{119}$ F. Giordano, ${ }^{119}$ G. Hanson, ${ }^{119}$ G. Y. Jeng, ${ }^{119, \text { uu }}$ H. Liu, ${ }^{119}$ O. R. Long, ${ }^{119}$ A. Luthra, ${ }^{119}$ H. Nguyen, ${ }^{119}$ S. Paramesvaran, ${ }^{119}$ J. Sturdy, ${ }^{119}$ S. Sumowidagdo, ${ }^{119}$ R. Wilken, ${ }^{119}$ S. Wimpenny, ${ }^{119}$ W. Andrews, ${ }^{120}$ J. G. Branson, ${ }^{120}$ G. B. Cerati, ${ }^{120}$ S. Cittolin, ${ }^{120}$ D. Evans, ${ }^{120}$ F. Golf, ${ }^{120}$ A. Holzner, ${ }^{120}$ R. Kelley ${ }^{120}$ M. Lebourgeois, ${ }^{120}$ J. Letts,${ }^{120}$ I. Macneill, ${ }^{120}$ B. Mangano, ${ }^{120}$ J. Muelmenstaedt, ${ }^{120}$ S. Padhi, ${ }^{120}$ C. Palmer,${ }^{120}$ G. Petrucciani, ${ }^{120}$ M. Pieri, ${ }^{120}$ R. Ranieri, ${ }^{120}$ M. Sani, ${ }^{120}$ V. Sharma, ${ }^{120}$ S. Simon, ${ }^{120}$ E. Sudano, ${ }^{120}$ M. Tadel, ${ }^{120}$ Y. Tu, ${ }^{120}$ A. Vartak, ${ }^{120}$ S. Wasserbaech, ${ }^{120, v v}$ F. Würthwein, ${ }^{120}$ A. Yagil, ${ }^{120}$ J. Yoo, ${ }^{120}$ D. Barge, ${ }^{121}$ R. Bellan,,${ }^{121}$ C. Campagnari, ${ }^{121}$ M. D' Alfonso, ${ }^{121}$ 
T. Danielson, ${ }^{121}$ K. Flowers, ${ }^{121}$ P. Geffert, ${ }^{121}$ J. Incandela, ${ }^{121}$ C. Justus, ${ }^{121}$ P. Kalavase, ${ }^{121}$ S. A. Koay, ${ }^{121}$ D. Kovalskyi, ${ }^{121, \mathrm{~b}}$ V. Krutelyov, ${ }^{121}$ S. Lowette, ${ }^{121}$ N. Mccoll, ${ }^{121}$ V. Pavlunin, ${ }^{121}$ F. Rebassoo, ${ }^{121}$ J. Ribnik, ${ }^{121}$ J. Richman, ${ }^{121}$ R. Rossin, ${ }^{121}$ D. Stuart, ${ }^{121}$ W. To, ${ }^{121}$ C. West, ${ }^{121}$ A. Apresyan, ${ }^{122}$ A. Bornheim, ${ }^{122}$ Y. Chen, ${ }^{122}$ E. Di Marco, ${ }^{122}$ J. Duarte, ${ }^{122}$ M. Gataullin, ${ }^{122}$ Y. Ma, ${ }^{122}$ A. Mott, ${ }^{122}$ H. B. Newman, ${ }^{122}$ C. Rogan, ${ }^{122}$ V. Timciuc, ${ }^{122}$ P. Traczyk, ${ }^{122}$ J. Veverka, ${ }^{122}$ R. Wilkinson, ${ }^{122}$ Y. Yang, ${ }^{122}$ R. Y. Zhu, ${ }^{122}$ B. Akgun, ${ }^{123}$ R. Carroll, ${ }^{123}$ T. Ferguson, ${ }^{123}$ Y. Iiyama, ${ }^{123}$ D. W. Jang, ${ }^{123}$ Y. F. Liu, ${ }^{123}$ M. Paulini,,${ }^{123}$ H. Vogel, ${ }^{123}$ I. Vorobiev, ${ }^{123}$ J. P. Cumalat, ${ }^{124}$ B. R. Drell, ${ }^{124}$ C. J. Edelmaier, ${ }^{124}$ W. T. Ford, ${ }^{124}$ A. Gaz, ${ }^{124}$ B. Heyburn, ${ }^{124}$ E. Luiggi Lopez, ${ }^{124}$ J. G. Smith, ${ }^{124}$ K. Stenson, ${ }^{124}$ K. A. Ulmer, ${ }^{124}$ S. R. Wagner, ${ }^{124}$ L. Agostino, ${ }^{125}$ J. Alexander, ${ }^{125}$ A. Chatterjee, ${ }^{125}$ N. Eggert, ${ }^{125}$ L. K. Gibbons, ${ }^{125}$ B. Heltsley, ${ }^{125}$ W. Hopkins, ${ }^{125}$ A. Khukhunaishvili,${ }^{125}$ B. Kreis, ${ }^{125}$ N. Mirman, ${ }^{125}$ G. Nicolas Kaufman, ${ }^{125}$ J. R. Patterson, ${ }^{125}$ A. Ryd, ${ }^{125}$ E. Salvati, ${ }^{125}$ W. Sun, ${ }^{125}$ W. D. Teo, ${ }^{125}$ J. Thom, ${ }^{125}$ J. Thompson, ${ }^{125}$ J. Vaughan, ${ }^{125}$

Y. Weng, ${ }^{125}$ L. Winstrom, ${ }^{125}$ P. Wittich, ${ }^{125}$ D. Winn, ${ }^{126}$ S. Abdullin, ${ }^{127}$ M. Albrow, ${ }^{127}$ J. Anderson, ${ }^{127}$

L. A. T. Bauerdick, ${ }^{127}$ A. Beretvas, ${ }^{127}$ J. Berryhill, ${ }^{127}$ P. C. Bhat, ${ }^{127}$ I. Bloch, ${ }^{127}$ K. Burkett, ${ }^{127}$ J. N. Butler, ${ }^{127}$ V. Chetluru, ${ }^{127}$ H. W. K. Cheung, ${ }^{127}$ F. Chlebana, ${ }^{127}$ V. D. Elvira, ${ }^{127}$ I. Fisk, ${ }^{127}$ J. Freeman, ${ }^{127}$ Y. Gao, ${ }^{127}$ D. Green, ${ }^{127}$ O. Gutsche, ${ }^{127}$ A. Hahn, ${ }^{127}$ J. Hanlon, ${ }^{127}$ R. M. Harris, ${ }^{127}$ J. Hirschauer, ${ }^{127}$ B. Hooberman, ${ }^{127}$ S. Jindariani, ${ }^{127}$ M. Johnson, ${ }^{127}$ U. Joshi, ${ }^{127}$ B. Kilminster, ${ }^{127}$ B. Klima, ${ }^{127}$ S. Kunori, ${ }^{127}$ S. Kwan, ${ }^{127}$ D. Lincoln, ${ }^{127}$ R. Lipton, ${ }^{127}$ L. Lueking, ${ }^{127}$ J. Lykken, ${ }^{127}$ K. Maeshima, ${ }^{127}$ J. M. Marraffino, ${ }^{127}$ S. Maruyama, ${ }^{127}$ D. Mason, ${ }^{127}$ P. McBride, ${ }^{127}$ K. Mishra, ${ }^{127}$ S. Mrenna, ${ }^{127}$ Y. Musienko, ${ }^{127, w w}$ C. Newman-Holmes, ${ }^{127}$ V. O’Dell, ${ }^{127}$ O. Prokofyev, ${ }^{127}$ E. Sexton-Kennedy, ${ }^{127}$ S. Sharma, ${ }^{127}$ W. J. Spalding, ${ }^{127}$ L. Spiegel, ${ }^{127}$ P. Tan, ${ }^{127}$ L. Taylor, ${ }^{127}$ S. Tkaczyk, ${ }^{127}$ N. V. Tran, ${ }^{127}$ L. Uplegger, ${ }^{127}$ E. W. Vaandering, ${ }^{127}$ R. Vidal, ${ }^{127}$ J. Whitmore, ${ }^{127}$ W. Wu,${ }^{127}$ F. Yang, ${ }^{127}$ F. Yumiceva, ${ }^{127}$ J. C. Yun, ${ }^{127}$ D. Acosta, ${ }^{128}$ P. Avery, ${ }^{128}$ D. Bourilkov, ${ }^{128}$ M. Chen, ${ }^{128}$ S. Das, ${ }^{128}$ M. De Gruttola, ${ }^{128}$ G. P. Di Giovanni, ${ }^{128}$ D. Dobur, ${ }^{128}$ A. Drozdetskiy, ${ }^{128}$ R. D. Field, ${ }^{128}$ M. Fisher, ${ }^{128}$ Y. Fu, ${ }^{128}$ I. K. Furic ${ }^{128}$ J. Gartner, ${ }^{128}$ J. Hugon, ${ }^{128}$ B. Kim, ${ }^{128}$ J. Konigsberg, ${ }^{128}$ A. Korytov, ${ }^{128}$ A. Kropivnitskaya, ${ }^{128}$ T. Kypreos, ${ }^{128}$ J. F. Low, ${ }^{128}$ K. Matchev, ${ }^{128}$ P. Milenovic, ${ }^{128, \mathrm{xx}}$ G. Mitselmakher, ${ }^{128}$ L. Muniz, ${ }^{128}$ R. Remington, ${ }^{128}$ A. Rinkevicius, ${ }^{128}$ P. Sellers, ${ }^{128}$ N. Skhirtladze, ${ }^{128}$ M. Snowball, ${ }^{128}$ J. Yelton, ${ }^{128}$ M. Zakaria, ${ }^{128}$ V. Gaultney, ${ }^{129}$ L. M. Lebolo, ${ }^{129}$ S. Linn, ${ }^{129}$ P. Markowitz, ${ }^{129}$ G. Martinez, ${ }^{129}$ J. L. Rodriguez, ${ }^{129}$ T. Adams, ${ }^{130}$ A. Askew, ${ }^{130}$ J. Bochenek, ${ }^{130}$ J. Chen, ${ }^{130}$ B. Diamond, ${ }^{130}$ S. V. Gleyzer, ${ }^{130}$ J. Haas, ${ }^{130}$ S. Hagopian, ${ }^{130}$ V. Hagopian, ${ }^{130}$ M. Jenkins, ${ }^{130}$ K. F. Johnson, ${ }^{130}$ H. Prosper, ${ }^{130}$ V. Veeraraghavan, ${ }^{130}$ M. Weinberg, ${ }^{130}$ M. M. Baarmand, ${ }^{131}$ B. Dorney, ${ }^{131}$ M. Hohlmann, ${ }^{131}$ H. Kalakhety, ${ }^{131}$ I. Vodopiyanov, ${ }^{131}$ M. R. Adams, ${ }^{132}$ I. M. Anghel, ${ }^{132}$ L. Apanasevich, ${ }^{132}$ Y. Bai, ${ }^{132}$ V.E. Bazterra, ${ }^{132}$ R. R. Betts, ${ }^{132}$ J. Callner, ${ }^{132}$ R. Cavanaugh,,${ }^{132}$ C. Dragoiu, ${ }^{132}$ O. Evdokimov, ${ }^{132}$ E. J. Garcia-Solis, ${ }^{132}$ L. Gauthier, ${ }^{132}$ C. E. Gerber, ${ }^{132}$ D. J. Hofman, ${ }^{132}$ S. Khalatyan ${ }^{132}$ F. Lacroix ${ }^{132}$ M. Malek, ${ }^{132}$ C. O’Brien, ${ }^{132}$ C. Silkworth, ${ }^{132}$ D. Strom,,${ }^{132}$ N. Varelas, ${ }^{132}$ U. Akgun, ${ }^{133}$ E. A. Albayrak, ${ }^{133}$ B. Bilki, ${ }^{133, \text { yy }}$ K. Chung, ${ }^{133}$ W. Clarida, ${ }^{133}$ F. Duru, ${ }^{133}$ S. Griffiths, ${ }^{133}$ C. K. Lae, ${ }^{133}$ J.-P. Merlo, ${ }^{133}$ H. Mermerkaya, ${ }^{133, z z}$ A. Mestvirishvili, ${ }^{133}$ A. Moeller, ${ }^{133}$ J. Nachtman, ${ }^{133}$ C. R. Newsom, ${ }^{133}$ E. Norbeck, ${ }^{133}$ J. Olson, ${ }^{133}$ Y. Onel, ${ }^{133}$ F. Ozok, ${ }^{133}$ S. Sen, ${ }^{133}$ E. Tiras, ${ }^{133}$ J. Wetzel,,${ }^{133}$ T. Yetkin, ${ }^{133}$ K. Yi, ${ }^{133}$ B. A. Barnett, ${ }^{134}$ B. Blumenfeld, ${ }^{134}$ S. Bolognesi, ${ }^{134}$ D. Fehling, ${ }^{134}$ G. Giurgiu, ${ }^{134}$ A. V. Gritsan, ${ }^{134}$ Z. J. Guo, ${ }^{134}$ G. Hu, ${ }^{134}$ P. Maksimovic, ${ }^{134}$ S. Rappoccio, ${ }^{134}$ M. Swartz, ${ }^{134}$ A. Whitbeck, ${ }^{134}$ P. Baringer, ${ }^{135}$ A. Bean, ${ }^{135}$ G. Benelli, ${ }^{135}$ O. Grachov, ${ }^{135}$ R. P. Kenny Iii, ${ }^{135}$ M. Murray, ${ }^{135}$ D. Noonan, ${ }^{135}$ V. Radicci, ${ }^{135}$ S. Sanders, ${ }^{135}$ R. Stringer, ${ }^{135}$ G. Tinti, ${ }^{135}$ J. S. Wood, ${ }^{135}$ V. Zhukova, ${ }^{135}$ A. F. Barfuss, ${ }^{136}$ T. Bolton, ${ }^{136}$ I. Chakaberia, ${ }^{136}$ A. Ivanov, ${ }^{136}$ S. Khalil, ${ }^{136}$ M. Makouski, ${ }^{136}$ Y. Maravin, ${ }^{136}$ S. Shrestha, ${ }^{136}$ I. Svintradze, ${ }^{136}$ J. Gronberg, ${ }^{137}$ D. Lange,${ }^{137}$ D. Wright, ${ }^{137}$ A. Baden, ${ }^{138}$ M. Boutemeur, ${ }^{138}$ B. Calvert, ${ }^{138}$ S. C. Eno, ${ }^{138}$ J. A. Gomez, ${ }^{138}$ N. J. Hadley, ${ }^{138}$ R. G. Kellogg, ${ }^{138}$ M. Kirn, ${ }^{138}$ T. Kolberg, ${ }^{138}$ Y. Lu, ${ }^{138}$ M. Marionneau, ${ }^{138}$ A. C. Mignerey, ${ }^{138}$ A. Peterman, ${ }^{138}$ K. Rossato, ${ }^{138}$ A. Skuja, ${ }^{138}$ J. Temple, ${ }^{138}$ M. B. Tonjes, ${ }^{138}$ S. C. Tonwar, ${ }^{138}$ E. Twedt, ${ }^{138}$ G. Bauer, ${ }^{139}$ J. Bendavid, ${ }^{139}$ W. Busza, ${ }^{139}$ E. Butz ${ }^{139}$ I. A. Cali, ${ }^{139}$ M. Chan, ${ }^{139}$ V. Dutta, ${ }^{139}$ G. Gomez Ceballos, ${ }^{139}$ M. Goncharov, ${ }^{139}$ K. A. Hahn, ${ }^{139}$ Y. Kim, ${ }^{139}$ M. Klute, ${ }^{139}$ Y.-J. Lee, ${ }^{139}$ W. Li, ${ }^{139}$ P. D. Luckey, ${ }^{139}$ T. Ma, ${ }^{139}$ S. Nahn, ${ }^{139}$ C. Paus,${ }^{139}$ D. Ralph, ${ }^{139}$ C. Roland, ${ }^{139}$ G. Roland, ${ }^{139}$ M. Rudolph, ${ }^{139}$ G. S. F. Stephans, ${ }^{139}$ F. Stöckli, ${ }^{139}$ K. Sumorok, ${ }^{139}$ K. Sung, ${ }^{139}$ D. Velicanu, ${ }^{139}$ E. A. Wenger, ${ }^{139}$ R. Wolf, ${ }^{139}$ B. Wyslouch, ${ }^{139}$ S. Xie, ${ }^{139}$ M. Yang, ${ }^{139}$ Y. Yilmaz, ${ }^{139}$ A. S. Yoon, ${ }^{139}$ M. Zanetti, ${ }^{139}$ S. I. Cooper, ${ }^{140}$ P. Cushman, ${ }^{140}$ B. Dahmes, ${ }^{140}$ A. De Benedetti, ${ }^{140}$ G. Franzoni, ${ }^{140}$ A. Gude,${ }^{140}$ J. Haupt, ${ }^{140}$ S. C. Kao, ${ }^{140}$ K. Klapoetke, ${ }^{140}$ Y. Kubota, ${ }^{140}$ J. Mans, ${ }^{140}$ N. Pastika, ${ }^{140}$ R. Rusack,${ }^{140}$ M. Sasseville, ${ }^{140}$ A. Singovsky, ${ }_{140}$ N. Tambe, ${ }^{140}$ J. Turkewitz, ${ }^{140}$ L. M. Cremaldi, ${ }^{141}$ R. Kroeger, ${ }^{141}$ L. Perera, ${ }^{141}$ R. Rahmat, ${ }^{141}$ D. A. Sanders, ${ }^{141}$ E. Avdeeva, ${ }^{142}$ K. Bloom, ${ }^{142}$ S. Bose, ${ }^{142}$ J. Butt, ${ }^{142}$ D. R. Claes, ${ }^{142}$ A. Dominguez, ${ }^{142}$ M. Eads,${ }^{142}$ P. Jindal, ${ }^{142}$ J. Keller, ${ }^{142}$ I. Kravchenko, ${ }^{142}$ J. Lazo-Flores, ${ }^{142}$ H. Malbouisson, ${ }^{142}$ S. Malik, ${ }^{142}$ G. R. Snow, ${ }^{142}$ 
U. Baur, ${ }^{143}$ A. Godshalk, ${ }^{143}$ I. Iashvili, ${ }^{143}$ S. Jain, ${ }^{143}$ A. Kharchilava, ${ }^{143}$ A. Kumar, ${ }^{143}$ S. P. Shipkowski, ${ }^{143}$ K. Smith, ${ }^{143}$ G. Alverson, ${ }^{144}$ E. Barberis,${ }^{144}$ D. Baumgartel, ${ }^{144}$ M. Chasco,${ }^{144}$ J. Haley, ${ }^{144}$ D. Trocino, ${ }^{144}$ D. Wood, ${ }^{144}$ J. Zhang, ${ }^{144}$ A. Anastassov, ${ }^{145}$ A. Kubik, ${ }^{145}$ N. Mucia,,${ }^{145}$ N. Odell,,${ }^{145}$ R. A. Ofierzynski, ${ }^{145}$ B. Pollack, ${ }^{145}$ A. Pozdnyakov, ${ }^{145}$ M. Schmitt, ${ }^{145}$ S. Stoynev, ${ }^{145}$ M. Velasco, ${ }^{145}$ S. Won, ${ }^{145}$ L. Antonelli,,${ }^{146}$ D. Berry, ${ }^{146}$ A. Brinkerhoff, ${ }^{146}$ M. Hildreth, ${ }^{146}$ C. Jessop, ${ }^{146}$ D. J. Karmgard, ${ }^{146}$ J. Kolb, ${ }^{146}$ K. Lannon, ${ }^{146}$ W. Luo, ${ }^{146}$ S. Lynch, ${ }^{146}$ N. Marinelli, ${ }^{146}$ D. M. Morse,${ }^{146}$ T. Pearson, ${ }^{146}$ R. Ruchti, ${ }^{146}$ J. Slaunwhite, ${ }^{146}$ N. Valls, ${ }^{146}$ J. Warchol, ${ }^{146}$ M. Wayne, ${ }^{146}$ M. Wolf, ${ }^{146}$ J. Ziegler, ${ }^{146}$ B. Bylsma, ${ }^{147}$ L. S. Durkin, ${ }^{147}$ C. Hill, ${ }^{147}$ R. Hughes,${ }^{147}$ P. Killewald, ${ }^{147}$ K. Kotov, ${ }^{147}$ T. Y. Ling, ${ }^{147}$ D. Puigh, ${ }^{147}$ M. Rodenburg, ${ }^{147}$ C. Vuosalo, ${ }^{147}$ G. Williams, ${ }^{147}$ B. L. Winer, ${ }^{147}$ N. Adam, ${ }^{148}$ E. Berry, ${ }^{148}$ P. Elmer, ${ }^{148}$ D. Gerbaudo, ${ }^{148}$ V. Halyo, ${ }^{148}$ P. Hebda, ${ }^{148}$ J. Hegeman, ${ }^{148}$ A. Hunt, ${ }^{148}$ E. Laird, ${ }^{148}$ D. Lopes Pegna, ${ }^{148}$ P. Lujan, ${ }^{148}$ D. Marlow, ${ }^{148}$ T. Medvedeva, ${ }^{148}$ M. Mooney, ${ }^{148} \mathrm{~J}$. Olsen, ${ }^{148}$ P. Piroué, ${ }^{148}$ X. Quan, ${ }^{148}$ A. Raval, ${ }^{148}$ H. Saka, ${ }^{148}$ D. Stickland, ${ }^{148}$ C. Tully, ${ }^{148}$ J. S. Werner, ${ }^{148}$ A. Zuranski, ${ }^{148}$ J. G. Acosta, ${ }^{149}$ X. T. Huang, ${ }^{149}$ A. Lopez,${ }^{149}$ H. Mendez ${ }^{149}$ S. Oliveros, ${ }^{149}$ J. E. Ramirez Vargas, ${ }^{149}$ A. Zatserklyaniy, ${ }^{149}$ E. Alagoz, ${ }^{150}$ V.E. Barnes, ${ }^{150}$ D. Benedetti, ${ }^{150}$ G. Bolla, ${ }^{150}$ D. Bortoletto, ${ }^{150}$ M. De Mattia,${ }^{150}$ A. Everett, ${ }^{150}$ Z. Hu, ${ }^{150}$ M. Jones, ${ }^{150}$ O. Koybasi, ${ }^{150}$ M. Kress, ${ }^{150}$ A. T. Laasanen, ${ }^{150}$ N. Leonardo, ${ }^{150}$ V. Maroussov, ${ }^{150}$ P. Merkel, ${ }^{150}$ D. H. Miller, ${ }^{150}$ N. Neumeister, ${ }^{150}$ I. Shipsey, ${ }^{150}$ D. Silvers, ${ }^{150}$ A. Svyatkovskiy, ${ }^{150}$ M. Vidal Marono, ${ }^{150}$ H. D. Yoo, ${ }^{150}$ J. Zablocki, ${ }^{150}$ Y. Zheng, ${ }^{150}$ S. Guragain, ${ }^{151}$ N. Parashar, ${ }^{151}$ A. Adair, ${ }^{152}$ C. Boulahouache, ${ }^{152}$ V. Cuplov, ${ }^{152}$ K. M. Ecklund, ${ }^{152}$ F. J. M. Geurts, ${ }^{152}$ B. P. Padley, ${ }^{152}$ R. Redjimi, ${ }^{152}$ J. Roberts,${ }^{152}$ J. Zabel, ${ }^{152}$ B. Betchart, ${ }^{153}$ A. Bodek, ${ }^{153}$ Y. S. Chung, ${ }^{153}$ R. Covarelli, ${ }^{153}$ P. de Barbaro, ${ }^{153}$ R. Demina, ${ }^{153}$ Y. Eshaq, ${ }^{153}$ A. Garcia-Bellido, ${ }^{153}$ P. Goldenzweig, ${ }^{153}$ Y. Gotra, ${ }^{153}$ J. Han,${ }^{153}$ A. Harel,${ }^{153}$ S. Korjenevski, ${ }^{153}$ D. C. Miner, ${ }^{153}$ D. Vishnevskiy, ${ }^{153}$ M. Zielinski, ${ }^{153}$ A. Bhatti, ${ }^{154}$ R. Ciesielski, ${ }^{154}$ L. Demortier, ${ }^{154}$ K. Goulianos, ${ }^{154}$ G. Lungu, ${ }^{154}$ S. Malik, ${ }^{154}$ C. Mesropian, ${ }^{154}$ S. Arora, ${ }^{155}$ A. Barker, ${ }^{155}$ J. P. Chou,,${ }^{155}$ C. Contreras-Campana, ${ }^{155}$

E. Contreras-Campana, ${ }^{155}$ D. Duggan, ${ }^{155}$ D. Ferencek, ${ }^{155}$ Y. Gershtein, ${ }^{155}$ R. Gray, ${ }^{155}$ E. Halkiadakis, ${ }^{155}$ D. Hidas, ${ }^{155}$ D. Hits, ${ }^{155}$ A. Lath, ${ }^{155}$ S. Panwalkar, ${ }^{155}$ M. Park, ${ }^{155}$ R. Patel, ${ }^{155}$ V. Rekovic, ${ }^{155}$ A. Richards, ${ }^{155}$ J. Robles, ${ }^{155}$ K. Rose, ${ }^{155}$ S. Salur, ${ }^{155}$ S. Schnetzer,${ }^{155}$ C. Seitz, ${ }^{155}$ S. Somalwar, ${ }^{155}$ R. Stone, ${ }^{155}$ S. Thomas, ${ }^{155}$ G. Cerizza, ${ }^{156}$ M. Hollingsworth, ${ }^{156}$ S. Spanier ${ }^{156}$ Z. C. Yang, ${ }^{156}$ A. York, ${ }^{156}$ R. Eusebi, ${ }^{157}$ W. Flanagan, ${ }^{157}$ J. Gilmore, ${ }^{157}$ T. Kamon, ${ }^{157 \text {,aaa }}$ V. Khotilovich, ${ }^{157}$ R. Montalvo, ${ }^{157}$ I. Osipenkov, ${ }^{157}$ Y. Pakhotin, ${ }^{157}$ A. Perloff, ${ }^{157}$ J. Roe, ${ }^{157}$ A. Safonov, ${ }^{157}$ T. Sakuma, ${ }^{157}$ S. Sengupta, ${ }^{157}$ I. Suarez, ${ }^{157}$ A. Tatarinov,${ }^{157}$ D. Toback, ${ }^{157}$ N. Akchurin, ${ }^{158}$ J. Damgov, ${ }^{158}$ P. R. Dudero, ${ }^{158}$ C. Jeong, ${ }^{158}$ K. Kovitanggoon, ${ }^{158}$ S. W. Lee, ${ }^{158}$ T. Libeiro, ${ }^{158}$ Y. Roh, ${ }^{158}$ I. Volobouev, ${ }^{158}$ E. Appelt, ${ }^{159}$ D. Engh, ${ }^{159}$ C. Florez, ${ }^{159}$ S. Greene, ${ }^{159}$ A. Gurrola,${ }^{159}$ W. Johns, ${ }^{159}$ P. Kurt, ${ }^{159}$ C. Maguire, ${ }^{159}$ A. Melo, ${ }^{159}$ P. Sheldon, ${ }^{159}$ B. Snook, ${ }^{159}$ S. Tuo, ${ }^{159}$ J. Velkovska, ${ }^{159}$ M. W. Arenton,${ }^{160}$ M. Balazs, ${ }^{160}$ S. Boutle,${ }^{160}$ B. Cox,${ }^{160}$ B. Francis, ${ }^{160}$ J. Goodell, ${ }^{160}$ R. Hirosky, ${ }^{160}$ A. Ledovskoy, ${ }^{160}$ C. Lin, ${ }^{160}$ C. Neu, ${ }^{160}$ J. Wood, ${ }^{160}$ R. Yohay, ${ }^{160}$ S. Gollapinni, ${ }^{161}$ R. Harr,${ }^{161}$ P.E. Karchin, ${ }^{161}$ C. Kottachchi Kankanamge Don, ${ }^{161}$ P. Lamichhane, ${ }^{161}$ A. Sakharov, ${ }^{161}$ M. Anderson, ${ }^{162}$ M. Bachtis, ${ }^{162}$ D. Belknap, ${ }^{162}$ L. Borrello, ${ }^{162}$ D. Carlsmith, ${ }^{162}$ M. Cepeda, ${ }^{162}$ S. Dasu, ${ }^{162}$ L. Gray, ${ }^{162}$ K. S. Grogg, ${ }^{162}$ M. Grothe, ${ }^{162}$ R. Hall-Wilton, ${ }^{162}$ M. Herndon, ${ }^{162}$ A. Hervé, ${ }^{162}$ P. Klabbers, ${ }^{162}$ J. Klukas, ${ }^{162}$ A. Lanaro, ${ }^{162}$ C. Lazaridis, ${ }^{162}$ J. Leonard, ${ }^{162}$ R. Loveless, ${ }^{162}$ A. Mohapatra, ${ }^{162}$ I. Ojalvo, ${ }^{162}$ G. A. Pierro, ${ }^{162}$ I. Ross, ${ }^{162}$ A. Savin, ${ }^{162}$ W. H. Smith, ${ }^{162}$ and J. Swanson ${ }^{162}$

\title{
(CMS Collaboration)
}

\author{
${ }^{1}$ Yerevan Physics Institute, Yerevan, Armenia \\ ${ }^{2}$ Institut für Hochenergiephysik der OeAW, Wien, Austria \\ ${ }^{3}$ National Centre for Particle and High Energy Physics, Minsk, Belarus \\ ${ }^{4}$ Universiteit Antwerpen, Antwerpen, Belgium \\ ${ }^{5}$ Vrije Universiteit Brussel, Brussel, Belgium \\ ${ }^{6}$ Université Libre de Bruxelles, Bruxelles, Belgium \\ ${ }^{7}$ Ghent University, Ghent, Belgium \\ ${ }^{8}$ Université Catholique de Louvain, Louvain-la-Neuve, Belgium \\ ${ }^{9}$ Université de Mons, Mons, Belgium \\ ${ }^{10}$ Centro Brasileiro de Pesquisas Fisicas, Rio de Janeiro, Brazil \\ ${ }^{11}$ Universidade do Estado do Rio de Janeiro, Rio de Janeiro, Brazil \\ ${ }^{12}$ Instituto de Fisica Teorica, Universidade Estadual Paulista, Sao Paulo, Brazil \\ ${ }^{13}$ Institute for Nuclear Research and Nuclear Energy, Sofia, Bulgaria \\ ${ }^{14}$ University of Sofia, Sofia, Bulgaria
}


${ }^{15}$ Institute of High Energy Physics, Beijing, China

${ }^{16}$ State Key Laboratory of Nuclear Physics and Technology, Peking University, Beijing, China

${ }^{17}$ Universidad de Los Andes, Bogota, Colombia

${ }^{18}$ Technical University of Split, Split, Croatia

${ }^{19}$ University of Split, Split, Croatia

${ }^{20}$ Institute Rudjer Boskovic, Zagreb, Croatia

${ }^{21}$ University of Cyprus, Nicosia, Cyprus

${ }^{22}$ Charles University, Prague, Czech Republic

${ }^{23}$ Academy of Scientific Research and Technology of the Arab Republic of Egypt,

Egyptian Network of High Energy Physics, Cairo, Egypt

${ }^{24}$ National Institute of Chemical Physics and Biophysics, Tallinn, Estonia

${ }^{25}$ Department of Physics, University of Helsinki, Helsinki, Finland

${ }^{26}$ Helsinki Institute of Physics, Helsinki, Finland

${ }^{27}$ Lappeenranta University of Technology, Lappeenranta, Finland

${ }^{28} \mathrm{DSM} / \mathrm{IRFU}$, CEA/Saclay, Gif-sur-Yvette, France

${ }^{29}$ Laboratoire Leprince-Ringuet, Ecole Polytechnique, IN2P3-CNRS, Palaiseau, France

${ }^{30}$ Institut Pluridisciplinaire Hubert Curien, Université de Strasbourg, Université de Haute-Alsace Mulhouse, CNRS/IN2P3, Strasbourg, France

${ }^{31}$ Centre de Calcul de l'Institut National de Physique Nucleaire et de Physique des Particules (IN2P3), Villeurbanne, France

${ }^{32}$ Université de Lyon, Université Claude Bernard Lyon 1, CNRS-IN2P3, Institut de Physique Nucléaire de Lyon, Villeurbanne, France

${ }^{33}$ Institute of High Energy Physics and Informatization, Tbilisi State University, Tbilisi, Georgia

${ }^{34}$ RWTH Aachen University, I. Physikalisches Institut, Aachen, Germany

${ }^{35}$ RWTH Aachen University, III. Physikalisches Institut A, Aachen, Germany

${ }^{36}$ RWTH Aachen University, III. Physikalisches Institut B, Aachen, Germany

${ }^{37}$ Deutsches Elektronen-Synchrotron, Hamburg, Germany

${ }^{38}$ University of Hamburg, Hamburg, Germany

${ }^{39}$ Institut für Experimentelle Kernphysik, Karlsruhe, Germany

${ }^{40}$ Institute of Nuclear Physics “Demokritos,” Aghia Paraskevi, Greece

${ }^{41}$ University of Athens, Athens, Greece

${ }^{42}$ University of Ioánnina, Ioánnina, Greece

${ }^{43}$ KFKI Research Institute for Particle and Nuclear Physics, Budapest, Hungary

${ }^{44}$ Institute of Nuclear Research ATOMKI, Debrecen, Hungary

${ }^{45}$ University of Debrecen, Debrecen, Hungary

${ }^{46}$ Panjab University, Chandigarh, India

${ }^{47}$ University of Delhi, Delhi, India

${ }^{48}$ Saha Institute of Nuclear Physics, Kolkata, India

${ }^{49}$ Bhabha Atomic Research Centre, Mumbai, India

${ }^{50}$ Tata Institute of Fundamental Research-EHEP, Mumbai, India

${ }^{51}$ Tata Institute of Fundamental Research-HECR, Mumbai, India

${ }^{52}$ Institute for Research in Fundamental Sciences (IPM), Tehran, Iran

${ }^{53}$ INFN Sezione di Bari, Bari, Italy

${ }^{53 \mathrm{~b}}$ Università di Bari, Bari, Italy

${ }^{53 c}$ Politecnico di Bari, Bari, Italy

${ }^{54 a}$ INFN Sezione di Bologna, Bologna, Italy

${ }^{54 \mathrm{~b}}$ Università di Bologna, Bologna, Italy

${ }^{55}$ aNFN Sezione di Catania, Catania, Italy

${ }^{55 b}$ Università di Catania, Catania, Italy

${ }^{56 \mathrm{a}}$ INFN Sezione di Firenze, Firenze, Italy

${ }^{56 \mathrm{~b}}$ Università di Firenze, Firenze, Italy

${ }^{57}$ INFN Laboratori Nazionali di Frascati, Frascati, Italy

${ }^{58}$ INFN Sezione di Genova, Genova, Italy

${ }^{59 a}$ INFN Sezione di Milano-Bicocca, Milano, Italy

${ }^{59 \mathrm{~b}}$ Università di Milano-Bicocca, Milano, Italy

${ }^{60 a}$ INFN Sezione di Napoli, Napoli, Italy

${ }^{60 \mathrm{~b}}$ Università di Napoli “Federico II,” Napoli, Italy

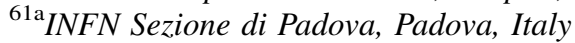

${ }^{61 \mathrm{~b}}$ Università di Padova, Padova, Italy

${ }^{61 \mathrm{c} U n i v e r s i t a ̀ ~ d i ~ T r e n t o ~(T r e n t o), ~ P a d o v a, ~ I t a l y ~}$

${ }^{62 a}$ INFN Sezione di Pavia, Pavia, Italy

${ }^{62 \mathrm{~b}}$ Università di Pavia, Pavia, Italy

${ }^{63 a}$ INFN Sezione di Perugia, Perugia, Italy 
${ }^{63 \mathrm{~b}}$ Università di Perugia, Perugia, Italy

${ }^{64 \mathrm{a}}$ INFN Sezione di Pisa, Pisa, Italy

${ }^{64 \mathrm{~b}}$ Università di Pisa, Pisa, Italy

${ }^{64 \mathrm{c}}$ Scuola Normale Superiore di Pisa, Pisa, Italy

${ }^{65 a}$ INFN Sezione di Roma, Roma, Italy

${ }^{65 \mathrm{~b}}$ Università di Roma "La Sapienza," Roma, Italy

${ }^{66 \mathrm{a}}$ INFN Sezione di Torino, Torino, Italy

${ }^{66 \mathrm{~b}}$ Università di Torino, Torino, Italy

${ }^{66 \mathrm{c}}$ Università del Piemonte Orientale (Novara), Torino, Italy

${ }^{67 \mathrm{a} I N F N}$ Sezione di Trieste, Trieste, Italy

${ }^{67 \mathrm{~b}}$ Università di Trieste, Trieste, Italy

${ }^{68}$ Kangwon National University, Chunchon, Korea

${ }^{69}$ Kyungpook National University, Daegu, Korea

${ }^{70}$ Chonnam National University, Institute for Universe and Elementary Particles, Kwangju, Korea

${ }^{71}$ Konkuk University, Seoul, Korea

${ }^{72}$ Korea University, Seoul, Korea

${ }^{73}$ University of Seoul, Seoul, Korea

${ }^{74}$ Sungkyunkwan University, Suwon, Korea

${ }^{75}$ Vilnius University, Vilnius, Lithuania

${ }^{76}$ Centro de Investigacion y de Estudios Avanzados del IPN, Mexico City, Mexico

${ }^{77}$ Universidad Iberoamericana, Mexico City, Mexico

${ }^{78}$ Benemerita Universidad Autonoma de Puebla, Puebla, Mexico

${ }^{79}$ Universidad Autónoma de San Luis Potosí, San Luis Potosí, Mexico

${ }^{80}$ University of Auckland, Auckland, New Zealand

${ }^{81}$ University of Canterbury, Christchurch, New Zealand

${ }^{82}$ National Centre for Physics, Quaid-I-Azam University, Islamabad, Pakistan

${ }^{83}$ Institute of Experimental Physics, Faculty of Physics, University of Warsaw, Warsaw, Poland

${ }^{84}$ Soltan Institute for Nuclear Studies, Warsaw, Poland

${ }^{85}$ Laboratório de Instrumentação e Física Experimental de Partículas, Lisboa, Portugal

${ }^{86}$ Joint Institute for Nuclear Research, Dubna, Russia

${ }^{87}$ Petersburg Nuclear Physics Institute, Gatchina (St Petersburg), Russia

${ }^{88}$ Institute for Nuclear Research, Moscow, Russia

${ }^{89}$ Institute for Theoretical and Experimental Physics, Moscow, Russia

${ }^{90}$ Moscow State University, Moscow, Russia

${ }^{91}$ P. N. Lebedev Physical Institute, Moscow, Russia

${ }^{92}$ State Research Center of Russian Federation, Institute for High Energy Physics, Protvino, Russia

${ }^{93}$ University of Belgrade, Faculty of Physics and Vinca Institute of Nuclear Sciences, Belgrade, Serbia

${ }^{94}$ Centro de Investigaciones Energéticas Medioambientales y Tecnológicas (CIEMAT), Madrid, Spain

${ }^{95}$ Universidad Autónoma de Madrid, Madrid, Spain

${ }^{96}$ Universidad de Oviedo, Oviedo, Spain

${ }^{97}$ Instituto de Física de Cantabria (IFCA), CSIC-Universidad de Cantabria, Santander, Spain

${ }^{98}$ CERN, European Organization for Nuclear Research, Geneva, Switzerland

${ }^{99}$ Paul Scherrer Institut, Villigen, Switzerland

${ }^{100}$ Institute for Particle Physics, ETH Zurich, Zurich, Switzerland

${ }^{101}$ Universität Zürich, Zurich, Switzerland

${ }^{102}$ National Central University, Chung-Li, Taiwan

${ }^{103}$ National Taiwan University (NTU), Taipei, Taiwan

${ }^{104}$ Cukurova University, Adana, Turkey

${ }^{105}$ Middle East Technical University, Physics Department, Ankara, Turkey

${ }^{106}$ Bogazici University, Istanbul, Turkey

${ }^{107}$ Istanbul Technical University, Istanbul, Turkey

${ }^{108}$ National Scientific Center, Kharkov Institute of Physics and Technology, Kharkov, Ukraine

${ }^{109}$ University of Bristol, Bristol, United Kingdom

${ }^{110}$ Rutherford Appleton Laboratory, Didcot, United Kingdom

${ }^{111}$ Imperial College, London, United Kingdom

${ }^{112}$ Brunel University, Uxbridge, United Kingdom

${ }^{113}$ Baylor University, Waco, Texas, USA

${ }^{114}$ The University of Alabama, Tuscaloosa, Alabama, USA

${ }^{115}$ Boston University, Boston, Massachusetts, USA

${ }^{116}$ Brown University, Providence, Rhode Island, USA

${ }^{117}$ University of California, Davis, Davis, California, USA 
${ }^{118}$ University of California, Los Angeles, Los Angeles, California, USA

${ }^{119}$ University of California, Riverside, Riverside, California, USA

${ }^{120}$ University of California, San Diego, La Jolla, California, USA

${ }^{121}$ University of California, Santa Barbara, Santa Barbara, California, USA

${ }^{122}$ California Institute of Technology, Pasadena, California, USA

${ }^{123}$ Carnegie Mellon University, Pittsburgh, Pennsylvania, USA

${ }^{124}$ University of Colorado at Boulder, Boulder, Colorado, USA ${ }^{125}$ Cornell University, Ithaca, New York, USA

${ }^{126}$ Fairfield University, Fairfield, Connecticut, USA

${ }^{127}$ Fermi National Accelerator Laboratory, Batavia, Illinois, USA

${ }^{128}$ University of Florida, Gainesville, Florida, USA

${ }^{129}$ Florida International University, Miami, Florida, USA

${ }^{130}$ Florida State University, Tallahassee, Florida, USA

${ }^{131}$ Florida Institute of Technology, Melbourne, Florida, USA

${ }^{132}$ University of Illinois at Chicago (UIC), Chicago, Illinois, USA

${ }^{133}$ The University of Iowa, Iowa City, Iowa, USA

${ }^{134}$ Johns Hopkins University, Baltimore, Maryland, USA

${ }^{135}$ The University of Kansas, Lawrence, Kansas, USA

${ }^{136}$ Kansas State University, Manhattan, Kansas, USA

${ }^{137}$ Lawrence Livermore National Laboratory, Livermore, California, USA

${ }^{138}$ University of Maryland, College Park, Maryland, USA

${ }^{139}$ Massachusetts Institute of Technology, Cambridge, Massachusetts, USA

${ }^{140}$ University of Minnesota, Minneapolis, Minnesota, USA

${ }^{141}$ University of Mississippi, University, Mississippi, USA

${ }^{142}$ University of Nebraska-Lincoln, Lincoln, Nebraska, USA

${ }^{143}$ State University of New York at Buffalo, Buffalo, New York, USA

${ }^{144}$ Northeastern University, Boston, Massachusetts, USA

${ }^{145}$ Northwestern University, Evanston, Illinois, USA

${ }^{146}$ University of Notre Dame, Notre Dame, Indiana, USA

${ }^{147}$ The Ohio State University, Columbus, Ohio, USA

${ }^{148}$ Princeton University, Princeton, New Jersey, USA

${ }^{149}$ University of Puerto Rico, Mayaguez, Puerto Rico, USA

${ }^{150}$ Purdue University, West Lafayette, Indiana, USA

${ }^{151}$ Purdue University Calumet, Hammond, Indiana, USA

${ }^{152}$ Rice University, Houston, Texas, USA

${ }^{153}$ University of Rochester, Rochester, New York, USA

${ }^{154}$ The Rockefeller University, New York, New York, USA

${ }^{155}$ Rutgers, the State University of New Jersey, Piscataway, New Jersey, USA

${ }^{156}$ University of Tennessee, Knoxville, Tennessee, USA

${ }^{157}$ Texas A\&M University, College Station, Texas, USA

${ }^{158}$ Texas Tech University, Lubbock, Texas, USA

${ }^{159}$ Vanderbilt University, Nashville, Tennessee, USA

${ }^{160}$ University of Virginia, Charlottesville, Virginia, USA

${ }^{161}$ Wayne State University, Detroit, Michigan, USA

${ }^{162}$ University of Wisconsin, Madison, Wisconsin, USA

${ }^{\mathrm{a}}$ Deceased.

${ }^{\mathrm{b}}$ Also at CERN, European Organization for Nuclear Research, Geneva, Switzerland.

${ }^{\mathrm{c}}$ Also at National Institute of Chemical Physics and Biophysics, Tallinn, Estonia.

${ }^{\mathrm{d}}$ Also at Universidade Federal do ABC, Santo Andre, Brazil.

${ }^{\mathrm{e}}$ Also at California Institute of Technology, Pasadena, CA, USA.

${ }_{\mathrm{f}}^{\mathrm{f}}$ Also at Laboratoire Leprince-Ringuet, Ecole Polytechnique, IN2P3-CNRS, Palaiseau, France.

${ }^{\mathrm{g}}$ Also at Suez Canal University, Suez, Egypt.

${ }^{\mathrm{h}}$ Also at Cairo University, Cairo, Egypt.

${ }^{i}$ Also at British University, Cairo, Egypt.

${ }^{\mathrm{j}}$ Also at Fayoum University, El-Fayoum, Egypt.

${ }^{\mathrm{k}}$ Also at Soltan Institute for Nuclear Studies, Warsaw, Poland.

${ }^{1}$ Also at Université de Haute-Alsace, Mulhouse, France.

${ }^{\mathrm{m}}$ Also at Moscow State University, Moscow, Russia. 
${ }^{\mathrm{n}}$ Also at Brandenburg University of Technology, Cottbus, Germany.

${ }^{\circ}$ Also at Institute of Nuclear Research ATOMKI, Debrecen, Hungary.

${ }^{\mathrm{p}}$ Also at Eötvös Loránd University, Budapest, Hungary.

${ }^{\mathrm{q}}$ Also at Tata Institute of Fundamental Research - HECR, Mumbai, India.

${ }^{\mathrm{r}}$ Also at University of Visva-Bharati, Santiniketan, India.

${ }^{\text {s} A l s o ~ a t ~ S h a r i f ~ U n i v e r s i t y ~ o f ~ T e c h n o l o g y, ~ T e h r a n, ~ I r a n . ~}$

${ }^{\mathrm{t}}$ Also at Isfahan University of Technology, Isfahan, Iran.

${ }^{u}$ Also at Shiraz University, Shiraz, Iran.

${ }^{v}$ Also at Plasma Physics Research Center, Science and Research Branch, Islamic Azad University, Teheran, Iran.

${ }^{\mathrm{w}}$ Also at Facoltà Ingegneria Università di Roma, Roma, Italy.

${ }^{\mathrm{x}}$ Also at Università della Basilicata, Potenza, Italy.

${ }^{\mathrm{y}}$ Also at Università degli Studi Guglielmo Marconi, Roma, Italy.

${ }^{\mathrm{z}}$ Also at Università degli studi di Siena, Siena, Italy.

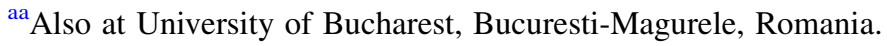

${ }^{\mathrm{bb}}$ Also at Faculty of Physics of University of Belgrade, Belgrade, Serbia.

${ }^{\mathrm{cc}}$ Also at University of Florida, Gainesville, FL, USA.

${ }^{\mathrm{dd}}$ Also at University of California, Los Angeles, Los Angeles, CA, USA.

${ }^{e e}$ Also at Scuola Normale e Sezione dell' INFN, Pisa, Italy.

${ }^{\mathrm{ff}}$ Also at INFN Sezione di Roma, Università di Roma "La Sapienza," Roma, Italy.

${ }^{\mathrm{gg}}$ Also at University of Athens, Athens, Greece.

${ }^{\text {hh }}$ Also at Rutherford Appleton Laboratory, Didcot, United Kingdom.

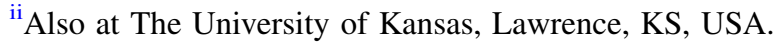

${ }^{\mathrm{jj}}$ Also at Paul Scherrer Institut, Villigen, Switzerland.

${ }^{\mathrm{kk}} \mathrm{Also}$ at Institute for Theoretical and Experimental Physics, Moscow, Russia.

${ }^{11}$ Also at Gaziosmanpasa University, Tokat, Turkey.

${ }^{\mathrm{mm}}$ Also at Adiyaman University, Adiyaman, Turkey.

${ }^{n n}$ Also at The University of Iowa, Iowa City, IA, USA.

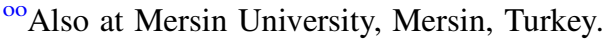

${ }^{\mathrm{pp}}$ Also at Kafkas University, Kars, Turkey.

${ }^{\mathrm{qq}}$ Also at Suleyman Demirel University, Isparta, Turkey.

${ }^{\mathrm{rr}}$ Also at Ege University, Izmir, Turkey.

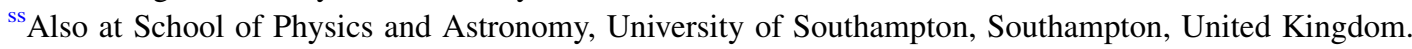

${ }^{\text {tt}}$ Also at INFN Sezione di Perugia, Università di Perugia, Perugia, Italy.

${ }^{u u}$ Also at University of Sydney, Sydney, Australia.

${ }^{\mathrm{vv}}$ Also at Utah Valley University, Orem, UT, USA.

${ }^{w w}$ Also at Institute for Nuclear Research, Moscow, Russia.

${ }^{\mathrm{xx}}$ Also at University of Belgrade, Faculty of Physics and Vinca Institute of Nuclear Sciences, Belgrade, Serbia.

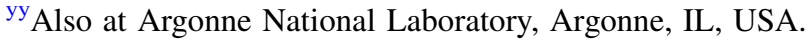

${ }^{\mathrm{zz}}$ Also at Erzincan University, Erzincan, Turkey.

${ }^{\text {aaa }}$ Also at Kyungpook National University, Daegu, Korea. 